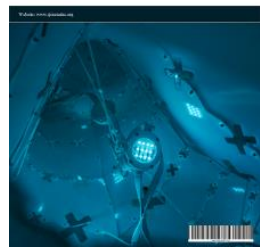

\title{
Bayesian Approaches for Poisson Distribution Parameter Estimation
}

\author{
Yadpirun Supharakonsakun ${ }^{1 *}$ \\ ${ }^{1}$ Faculty of Science and Technology, Phetchabun Rajabhat University,Phetchabun, 67000, Thailand
}

\begin{abstract}
The Bayesian approach, a non-classical estimation technique, is very widely used in statistical inference for real world situations. The parameter is considered to be a random variable, and knowledge of the prior distribution is used to update the parameter estimation. Herein, two Bayesian approaches for Poisson parameter estimation by deriving the posterior distribution under the squared error loss or quadratic loss functions are proposed. Their performances were compared with frequentist (maximum likelihood estimator) and Empirical Bayes approaches through Monte Carlo simulations. The mean square error was used as the test criterion for comparing the methods for point estimation; the smallest value indicates the best performing method with the estimated parameter value closest to the true parameter value. Coverage Probabilities (CPs) and average lengths (ALs) were obtained to evaluate the performances of the methods for constructing confidence intervals. The results reveal that the Bayesian approaches were excellent for point estimation when the true parameter value was small $(\theta=0.5,1$ and 2$)$. In the credible interval comparison, these methods obtained CP values close to the nominal 0.95 confidence level and the smallest ALs for large sample sizes $(n=50$ and 100$)$, when the true parameter value was small $(\theta=0.5,1$ and 2$)$
\end{abstract}

\section{Keywords:}

Bayesian Method;

Empirical Bayes Approach;

Poisson Distribution;

Maximum Likelihood Estimator;

Monte Carlo Simulation.

\section{Article History:}

$\begin{array}{llll}\text { Received: } & 02 & \text { July } & 2021 \\ \text { Revised: } & 12 & \text { August } & 2021 \\ \text { Accepted: } & 03 & \text { September } & 2021 \\ \text { Published: } & 01 & \text { October } & 2021\end{array}$

\section{1- Introduction}

The Poisson distribution plays an important role in the statistical analysis of count data. This type of data arises from situations in which there are several opportunities for the event of interest to occur, such as the number of customers calling a help center in a day, visitors to a net idol YouTube channel, patients infected with Covid-19 per day, and so on .Therefore, the Poisson distribution can be used to determine the probability of several events in a particular time period.

Various researchers have developed inference procedures for a Poisson distribution. Araveeporn [1] proposed inferential statistics for testing hypotheses by using the mean of a Poisson parameter estimator obtained via the maximum likelihood estimator (MLE), Markov Chain-Monte Carlo, and Bayesian approaches. Hassan et al. [2] investigated Bayesian and MLE estimators for a zero-truncated Poisson distribution. Bayesian estimators for a Poisson distribution using a natural conjugate prior [3] and under linex loss function [4-6] and different symmetric and asymmetric loss functions (squared error, linex, precautionary and general entropy) [7] have also been presented. As well as the Poisson distribution, the Bayesian technique for parameter estimation has been extended to the geometric distribution [8], binomial distribution [9], Pareto distribution [10], exponential distribution family [11, 12], double exponential distribution under symmetric and asymmetric loss functions [13], gamma distribution under generalized weighted loss

*CONTACT: yadpirun.suph@pcru.ac.th

DOI: http://dx.doi.org/10.28991/esj-2021-01310

(C) 2021 by the authors. Licensee ESJ, Italy. This is an open access article under the terms and conditions of the Creative Commons Attribution (CC-BY) license (https://creativecommons.org/licenses/by/4.0/). 
function [14], under precautionary loss function [15] and under entropy loss function [16], Weibull distribution [16], inverse Weibull distribution [18], Rayleigh distribution [19], delta-lognormal distribution [20-22], the Lomax distribution base on type-II censored data [23] and using a Uniform and Jeffery prior under different loss functions [24], and inverse Lomax distribution under progressive type-II censoring scheme [25]. The results mostly indicate that the Bayesian-based estimates using the different loss functions were close to the true values, thereby suggesting that this could be a useful approach for estimating parameters of interest in various situations.

The Empirical Bayesian (EB) approach introduced by Robbins [26] is an approximation of the more exact Bayesian approach for a certain sample size. EB is independent with a fixed but unknown parameter of the prior distribution. Estimating this parameter by analyzing the current data is the first step in the EB method. This is not a typical or pure Bayesian method since the parameter of the prior is specified and estimating the hyperprior must be carried out via a classical method such as MLE or the method of moments.

Many researchers have studied Bayesian and EB methods in various situations. Supharakonsaun and Jampachasri [27] introduced an EB estimator for a Poisson distribution by using an exponential distribution that is a special case of a gamma distribution under the squared-error loss function as the posterior marginal distribution; MLE was used to estimate its hyperparameter, which was extended for estimating the hyperparameter via a resampling technique. Mohammed [28] reported expected Bayesian and expected EB approaches for estimating the unknown parameter of a Poisson model under the squared-error loss function. The findings from these two studies indicate that Bayesian and EB estimators are more effective than classical estimators. The efficacy of EB has been compared with hierarchical Bayesian estimation for the unknown parameter of a Poisson distribution under the entropy loss function [29]; the expression of EB is simpler and it performed better than hierarchical Bayesian estimation.

Popular classical methods for estimating the hyperparameter of the prior distribution are MLE and the method of moments. Estimating the Poisson parameter via the Bayesian posterior distribution under Stein's method or the squarederror loss function and an EB estimator with a conjugate gamma prior showed that the MLE for hyperparameter estimation is better than the method of moments estimator for the EB approach [30]. Similarly, the case of a normal distribution with a conjugated normal-inverse-gamma prior was computed by Zhang et al. [31].

Approaches for estimating the parameter of a random variable can be grouped into 3 categories: classical, Bayesian, and empirical Bayesian (EB), which are all useful under different sets of circumstances. The Bayesian methods provide estimates of the unknown parameter by using a fixed informative prior distribution, are better than classical approaches for most situations involving loss functions. Therefore, estimating a Poisson parameter using Bayesian methods under different loss functions is of interest in the present study.

Our motivation for this study is to estimate the Poisson parameter by using MLE, Bayesian, and EB methods for both point and interval estimation. The main goal of the paper is focused the Bayesian estimation of Poisson parameter under the squared error and quadratic loss functions. Derivations of these approaches are covered in Section 2. The results of a simulation study are reported in Section 3. Finally, the conclusions based on our findings are presented in the last section.

\section{2- Estimation Methods for Mean of Poisson Distribution}

\section{2-1- The Maximum Likelihood Estimator (MLE)}

MLE is a simple method for constructing an estimator for the unknown parameter of a probability distribution by maximizing a likelihood function. It can be applied to a wide variety of statistical problems and provides a reasonable and excellent estimator for the parameter when the sample size is large.

Suppose $X_{1}, X_{2}, \ldots, X_{n}$ are random variables with a probability mass function from independent and identically distributed random variables from a Poisson distribution with parameter $\theta$. The probability function of $X_{i} ; i=1,2, \ldots, n$ denoted by $f\left(x_{i} \mid \theta\right)$ can be derived as follows:

$$
f\left(x_{i} \mid \theta\right)=\frac{e^{-\theta} \theta^{x_{i}}}{x_{i} !} ; x_{i}=0,1,2, \ldots ; \theta>0 \text {, }
$$

where $\theta$ is constant mean rate of event occur.

The joint probability mass function or product of $n$ terms is called likelihood function, which is defined as;

$$
L(\underline{X} \mid \theta)=\prod_{i=1}^{n} f\left(x_{i} \mid \theta\right)=\frac{e^{-n \theta} \theta^{\sum_{i=1}^{n} x_{i}}}{\prod_{i=1}^{n} x_{i} !} .
$$

In order to make the joint probability mass function to be a monotonic function, the logarithm is taken to the likelihood function. That is; 
$\ln L(\underline{X} \mid \theta)=-n \theta+\sum_{i=1}^{n} x_{i} \ln \theta-\ln \prod_{i=1}^{n} x_{i} !$.

From the logarithm of the likelihood function, the maximum of $\ln L(\underline{X} \mid \theta)$ occurs at the same value of $\theta$ as does the maximum of $L(\underline{X} \mid \theta)$. If $\ln L(\underline{X} \mid \theta)$ is differentiable in parameter $\theta$, the necessary conditions for the occurrence of a maximum is solved by applying;

$\frac{\partial \ln L(\underline{X} \mid \lambda)}{\partial \theta}=-n+\frac{\sum_{i=1}^{n} x_{i}}{\theta}=0$.

By the solving of necessary condition as mentioned above, we can obtain the MLE of $\theta$ as

$\hat{\theta}_{M L E}=\frac{\sum_{i=1}^{n} x_{i}}{n}=\bar{x}$.

Because we do not know if this is a maximum or minimum value, the second derivative of the estimator can be used to prove that the estimator is the maximum when the second derivative is less than 0 as follows:

$\frac{\partial^{2} \ln L(\underline{X} \mid \lambda)}{\partial \theta^{2}}=-\frac{\sum_{i=1}^{n} x_{i}}{\theta^{2}}<0$.

Therefore, the $M L E$ of $\theta$ is $\hat{\theta}_{M L E}=\bar{x}$.

\section{2-2-Bayesian Method}

To estimate the Bayesian estimator, the prior probability distribution under gamma prior is specified by using squared error and quadratic loss functions.

Let $X_{1}, X_{2}, \ldots, X_{n}$ be random variables from a Poisson distribution. The probability mass function of the random variable is given by $f\left(x_{i} \mid \theta\right)$ with the likelihood function $L(\theta)=\prod_{i=1}^{n} f\left(x_{i} \mid \theta\right)$. Consider the informative conjugate prior for $\theta$ is a Gamma distribution with parameters $a, b$ where $a$ is the shape parameter and $b$ is the scale parameter. It is given by,

$h(\theta \mid a, b)=\frac{b^{a}}{\Gamma(a)} \theta^{a-1} e^{-b \theta}, a, b, \theta>0$.

The posterior distribution for Bayesian procedure can be derived by considering the combining of the likelihood function (2) and the prior distribution (7) as follows:

$f(\underline{x} \mid \theta) \pi(\theta)=\frac{b^{a} e^{-(n+b) \theta} \cdot \theta^{\sum_{i=1}^{n} x_{i}+a-1}}{\prod_{i=1}^{n} x_{i} ! \Gamma(a)}$

The marginal probability density function of $\theta$ can be derived by integration of the combing of the likelihood function and the prior distribution. It can be derived as follows:

$$
\begin{aligned}
& \int_{0}^{\infty} f(\underline{x} \mid \theta) \pi(\theta) d \theta=\int_{0}^{\infty} \frac{b^{a} e^{-(n+b) \theta} \cdot \theta^{\sum_{i=1}^{n} x_{i}+a-1}}{\prod_{i=1}^{n} x_{i} ! \Gamma(a)} d \theta \\
& =\frac{b^{a} \Gamma\left(\sum_{i=1}^{n} x_{i}+a\right)}{\prod_{i=1}^{n} x_{i} ! \Gamma(a)(n+b) \sum_{i=1}^{n} x_{i}+a} \int_{0}^{\infty} \frac{(n+b) \sum_{i=1}^{n} x_{i}+a}{e^{-(n+b) \theta} \cdot \theta^{\sum_{i=1}^{n} x_{i}+a-1}} \Gamma_{\left(\sum_{i=1}^{n} x_{i}+a\right)} d \theta=\frac{b^{a} \Gamma\left(\sum_{i=1}^{n} x_{i}+a\right)}{\prod_{i=1}^{n} x_{i} ! \Gamma(a)(n+b) \sum_{i=1}^{n} x_{i}+a}
\end{aligned}
$$

Now, the posterior distribution of $\theta$ is derived by; 


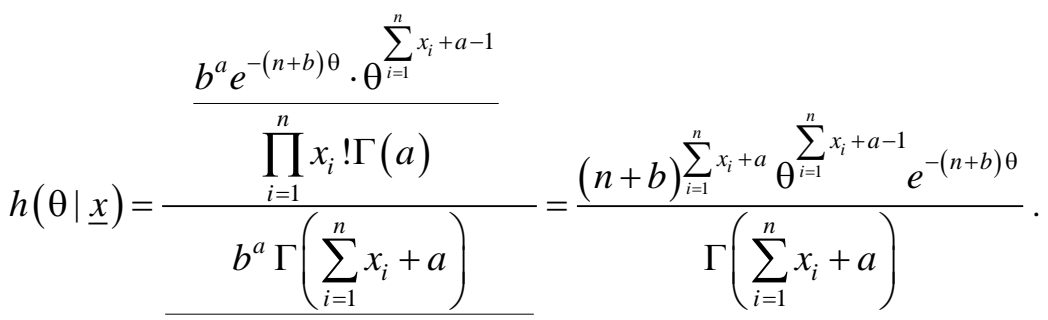

$$
\begin{aligned}
& \prod_{i=1}^{n} x_{i} ! \Gamma(a)(n+b) \sum_{i=1}^{n} x_{i}+a
\end{aligned}
$$

This implies that the posterior distribution can be written as;

$$
h(\theta \mid \underline{x})=\frac{(n+b) \sum_{i=1}^{n} x_{i}+a}{\Gamma\left(\sum_{i=1}^{n} x_{i}+a\right)} \theta^{\sum_{i=1}^{n} x_{i}+a-1} e^{-(n+b) \theta},
$$

which is a gamma distribution with parameters $\sum_{i=1}^{n} x_{i}+a$ and $n+b$. Hence,

$$
\theta \mid \underline{X} \sim \operatorname{Gamma}\left(\sum_{i=1}^{n} x_{i}+a, n+b\right) \text {. }
$$

\section{2-2-1- The Bayesian Estimator of Parameter $\theta$ for Squared Error (SE) Loss Function}

The Bayesian estimator for $\theta$ for the squared error loss function that is defined as;

$$
L(\hat{\theta} ; \theta)=(\hat{\theta}-\theta)^{2}
$$

The squared error loss function of the Bayesian estimator is the mean of the posterior distribution function, which can be derived as;

$$
\begin{aligned}
& \hat{\theta}_{B S}=E(\theta \mid \underline{x})
\end{aligned}
$$

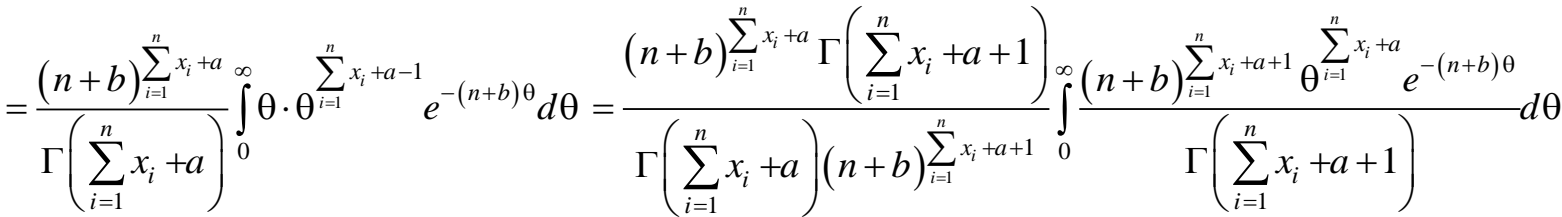

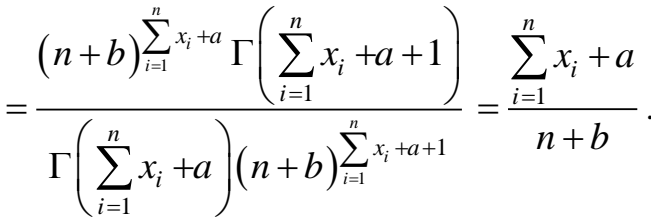

The Bayesian estimator of $\theta$ under the squared error loss function is shown by,

$\hat{\theta}_{B S}=\frac{\sum_{i=1}^{n} x_{i}+a}{n+b}$.

\section{2-2-2- The Bayesian Estimator of Parameter $\theta$ for Quadratic Loss (QL) Function}

The quadratic loss function which is a non-negative symmetric and continuous loss function of parameter $\theta$ and estimate of $\hat{\theta}$ for the Bayesian estimator is defined as [12];

$L(\hat{\theta} ; \theta)=\left(\frac{\hat{\theta}-\theta}{\theta}\right)^{2}$.

The Bayesian estimator under quadratic loss function of $\theta$ is obtained by deriving the following equation: 
$\frac{\partial}{\partial \hat{\theta}} \int L(\hat{\theta} ; \theta) h(\theta \mid \underline{x}) d \theta=0$.

The Bayesian estimator for parameter $\theta$ of the Poisson distribution under a quadratic loss function can be solved by;

$$
\begin{aligned}
& \frac{\partial}{\partial \hat{\theta}} \int\left(\frac{\hat{\theta}-\theta}{\theta}\right)^{2} \frac{(n+b) \sum_{i=1}^{n} x_{i}+a}{\Gamma\left(\sum_{i=1}^{n} x_{i}+a\right)} \theta^{\sum_{i=1}^{n} x_{i}+a-1} e^{-(n+b) \theta} d \theta=0 \\
& \int \frac{2(\hat{\theta}-\theta)}{\theta^{2}} \frac{(n+b) \sum_{i=1}^{n} x_{i}+a}{\Gamma\left(\sum_{i=1}^{n} x_{i}+a\right)} \theta^{\sum_{i=1}^{n} x_{i}+a-1} e^{-(n+b) \theta} d \theta=0 \\
& \frac{(n+b) \sum_{i=1}^{n} x_{i}+a}{\Gamma\left(\sum_{i=1}^{n} x_{i}+a\right)} \hat{\theta} \int \theta^{\left(\sum_{i=1}^{n} x_{i}+a-2\right)-1} e^{-(n+b) \theta} d \theta=\frac{(n+b) \sum_{i=1}^{n} x_{i}+a}{\Gamma\left(\sum_{i=1}^{n} x_{i}+a\right)} \int \theta^{\left(\sum_{i=1}^{n} x_{i}+a-1\right)-1} e^{-(n+b) \theta} d \theta \\
& \frac{(n+b) \sum_{i=1}^{n} x_{i}+a}{\Gamma\left(\sum_{i=1}^{n} x_{i}+a\right)} \hat{\theta} \frac{\Gamma\left(\sum_{i=1}^{n} x_{i}+a-2\right)}{(n+b) \sum_{i=1}^{n} x_{i}+a-2}=\frac{(n+b) \sum_{i=1}^{n} x_{i}+a}{\Gamma\left(\sum_{i=1}^{n} x_{i}+a\right)} \frac{\Gamma\left(\sum_{i=1}^{n} x_{i}+a-1\right)}{(n+b) \sum_{i=1}^{n} x_{i}+a-1} \\
& \hat{\theta}=\frac{\Gamma\left(\sum_{i=1}^{n} x_{i}+a-1\right)}{(n+b) \sum_{i=1}^{n} x_{i}+a-1} \frac{(n+b) \sum_{i=1}^{n} x_{i}+a-2}{\Gamma\left(\sum_{i=1}^{n} x_{i}+a-2\right)}=\frac{\sum_{i=1}^{n} x_{i}+a-2}{n+b}
\end{aligned}
$$

The Bayesian estimator of $\theta$ under a quadratic loss function is expressed as;

$$
\hat{\theta}_{Q L}=\frac{\sum_{i=1}^{n} x_{i}+a-2}{n+b} .
$$

\section{2-3- The Empirical Bayes (EB) Estimation}

The idea behind the EB method is different from the Bayesian approach .To estimate the true parameter value by using the Bayesian approach, the hyperparameter is assumed to be known or if unknown, prior information on it is available .The hyperparameter is independent of the observations.With the EB method, the first step of estimating the hyperparameter is by using the classical approach of assessing the observations, after which the hyperparameter is substituted by the estimation in the posterior distribution.

Let $X_{1}, X_{2}, \ldots, X_{n}$ be random variables from a Poisson distribution. The probability mass function of the random variable is given by (1):

$$
f\left(x_{i} \mid \theta\right)=\frac{e^{-\theta} \theta^{x_{i}}}{x_{i} !}, x_{i}=0,1,2, \ldots .
$$

Supharakonsakun and Jampachasri [27] proposed the EB estimator by studying the case of $\theta$ as ab exponential distribution denoted by $\theta \sim \operatorname{Exp}(\lambda)$. Hence, the prior distribution function of $\theta$ can be written as;

$$
\pi(\theta)=\lambda e^{-\lambda \theta} ; \theta, \lambda>0 .
$$

Subsequently, the posterior marginal distribution of $X$ can be solved by; 
$m\left(x_{i} \mid \theta\right)=\int_{0}^{\infty} f(x \mid \lambda) \pi(\theta) d \theta$.

This is derived as follows:

$$
\begin{aligned}
& m\left(x_{i} \mid \lambda\right)=\int_{0}^{\infty} \frac{e^{-\theta} \theta^{x_{i}}}{x_{i} !} \lambda e^{-\lambda \theta} d \theta=\frac{\lambda}{x_{i} !} \frac{\Gamma\left(x_{i}+1\right)}{(\lambda+1)^{x_{i}+1}} \int_{0}^{\infty} \frac{(\theta+1)^{x_{i}+1}}{\Gamma\left(x_{i}+1\right)} \theta^{\left(x_{i}+1\right)-1} e^{-(\lambda+1) \theta} d \theta=\frac{\lambda}{x_{i} !} \frac{\Gamma\left(x_{i}+1\right)}{(\lambda+1)^{x_{i}+1}} \\
& m\left(x_{i} \mid \lambda\right)=\frac{\lambda}{(\lambda+1)^{x_{i}+1}} ; x_{i}=0,1,2, \ldots .
\end{aligned}
$$

We now obtain the posterior marginal distribution of $X$ as a geometric distribution denoted by $X \mid \lambda \sim G E O\left(\frac{\lambda}{\lambda+1}\right)$, which can be rewritten in the form;

$$
m\left(x_{i} \mid \lambda\right)=\left(\frac{\lambda}{\lambda+1}\right)\left(1-\frac{\lambda}{\lambda+1}\right)^{x_{i}-1} ; x_{i}=1,2,3, \ldots .
$$

Hyperparameter $\lambda$ is estimated in the next step of the EB procedure. The $M L E$ is used to estimate $\lambda$ by considering the likelihood function of the posterior marginal distribution as follows:

$$
L(\underline{X} \mid \lambda)=\prod_{i=1}^{n} \frac{\lambda}{(\lambda+1)^{x_{i}+1}}=\frac{\lambda^{n}}{(\lambda+1)^{\sum_{i=1}^{n} x_{i}+n}} .
$$

The logarithm of the likelihood function is;

$$
\ln L(\underline{X} \mid \lambda)=n \ln \lambda-\left(\sum_{i=1}^{n} x_{i}+n\right) \ln (\lambda+1) .
$$

From the logarithm of the likelihood function, $M L E$ for $\lambda$ is solved by;

$$
\frac{\partial \ln L(\underline{X} \mid \lambda)}{\partial \lambda}=\frac{n}{\lambda}-\frac{\sum_{i=1}^{n} x_{i}+n}{\lambda+1}=0 .
$$

We obtain the $M L E$ of the hyperparameter $\lambda$ as;

$$
\hat{\lambda}_{M L E}=\frac{n}{\sum_{i=1}^{n} x_{i}}=\frac{1}{\bar{x}} \text {. }
$$

The posterior distribution of the EB procedure can be derived as the product of the likelihood function and the prior distribution as follows:

$$
f(\underline{x} \mid \theta) \pi(\theta)=\frac{\lambda e^{-(n+\lambda) \theta} \cdot \theta^{\sum_{i=1}^{n} x_{i}}}{\prod_{i=1}^{n} x_{i} !} .
$$

The integration of the product produces;

$$
\int_{0}^{\infty} f(\underline{x} \mid \theta) \pi(\theta) d \theta=\int_{0}^{\infty} \frac{\lambda e^{-(n+\lambda) \theta} \cdot \theta^{\sum_{i=1}^{n} x_{i}}}{\prod_{i=1}^{n} x_{i} !} d \theta=\frac{\lambda \Gamma\left(\sum_{i=1}^{n} x_{i}+1\right)}{\prod_{i=1}^{n} x_{i} !(n+\lambda) \sum_{i=1}^{n} x_{i}+1} \int_{0}^{\infty} \frac{(n+\lambda)_{i=1}^{n} x_{i}+1}{\Gamma\left(\sum_{i=1}^{n} x_{i}+1\right)} e^{-(n+\lambda) \theta} \cdot \theta^{\left(\sum_{i=1}^{n} x_{i}+1\right)-1} d \theta .
$$

Thus, 


$$
\int_{0}^{\infty} f(\underline{x} \mid \theta) \pi(\theta) d \theta=\frac{\lambda \Gamma\left(\sum_{i=1}^{n} x_{i}+1\right)}{\prod_{i=1}^{n} x_{i} !(n+\lambda) \sum_{i=1}^{n} x_{i}+1} .
$$

The posterior distribution of $\theta$ given $\underline{x}$ becomes;

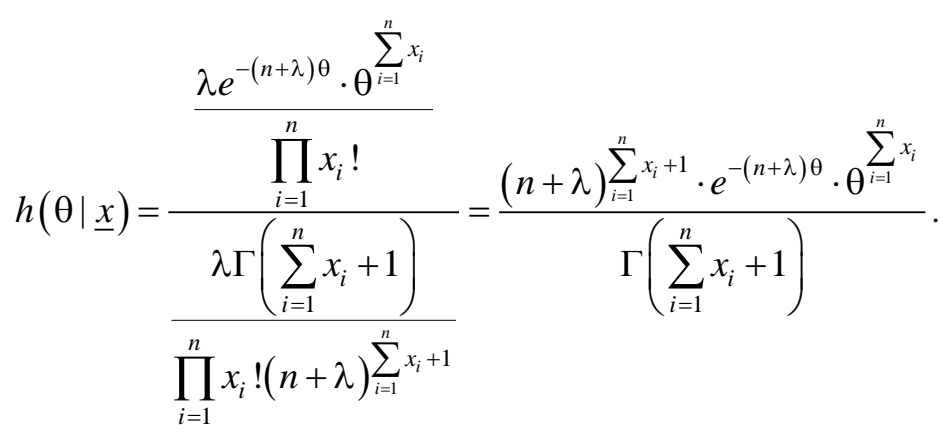

We now have the posterior distribution of $\underline{x}$ that is the Gamma distribution denoted by;

$\theta \mid \underline{X} \sim \operatorname{Gamma}\left(\sum_{i=1}^{n} x_{i}+1, n+\lambda\right)$.

After we have obtained the posterior distribution, we then substitute the hyperparameter $\lambda$ with estimator $\hat{\lambda}$ in the posterior distribution of $\underline{x}$. Hence, we obtain;

$\theta \mid \underline{X} \sim \operatorname{Gamma}\left(\sum_{i=1}^{n} x_{i}+1, n+\hat{\lambda}\right)$.

Therefore, the EB estimator of $\theta$ under the squared error loss function is given by;

$\hat{\theta}_{E B}=\frac{\sum_{i=1}^{n} x_{i}+1}{n+\hat{\lambda}}$.

Moreover, point estimation of hyperparameter is extended via a bootstrapping method for 1,000 iterations. Thus, there are two methods to estimate the true parameter $\theta$ using the EB procedure (EB and EB with bootstrappings).

\section{3- Simulation Results}

A simulation study with 5,000 replications was conducted to estimate the performance of point estimates and confidence intervals constructed with the 5 methods. For point estimation, the lowest mean-squared error (MSE) was the criterion used to identify the best-performing method. Besides, for comparing the confidence intervals constructed with them, coverage probabilities (CPs) and average lengths (Als) were calculated under the same conditions of varying hyperparameters $a$ and $b$ for the Bayesian approaches in the point estimation. A CP close to or greater than the nominal level of 0.95 and the shortest AL were used to identify the best-performing method in each case. The Monte Carlo simulation study is presented in Figure 1.

\section{3-1- Point Estimation}

The point estimation performances of the MLE, Bayesian under the squared-error loss function ( $\left.\mathrm{B}_{\mathrm{SL}}\right)$, Bayesian under quadratic loss function $\left(\mathrm{B}_{\mathrm{QL}}\right), \mathrm{EB}$, and $\mathrm{EB}$ with bootstrapping $\left(\mathrm{EB}_{\text {boot }}\right)$ methods for estimating the mean of a Poisson distribution were compared via simulation. Random samples were implemented to generate 5,000 sets from a Poisson distribution and 1,000 iterations for bootstrapping the hyperparameter estimation for $\mathrm{EB}_{\text {boot }}$ with various sample sizes $(n=5,10,15,20,30,50,100)$ and true parameter $\theta=0.5,1,2,5,10,20$ given arbitrary prior parameter $(a, b)=$ $(2,2),(2,4),(1,3.5)$ and $(4,4)$ for Bayesian methods under two different loss functions. The mean-squared error (MSE) is the criterion used to evaluate the performances of the parameter estimation methods, which is computed as

$M S E=\frac{\sum_{t=1}^{m}\left(\theta-\hat{\theta}_{t}\right)}{m}$.

The lowest MSE value means that the estimated value of $\theta$ is closest to its true value. 


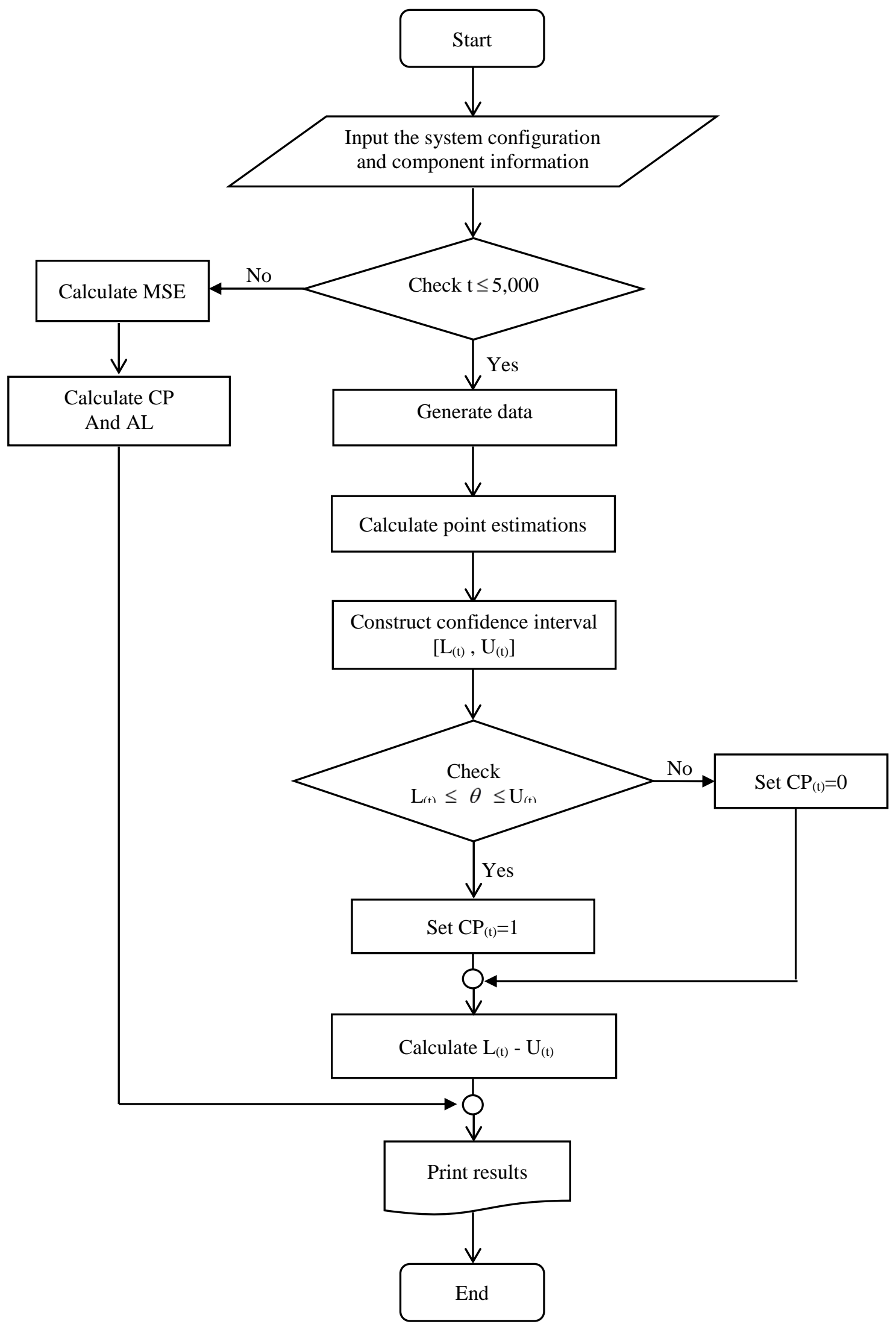

Figure 1. Monte Carlo simulation flowchart.

Table 1 reports the MSE values for point estimation using MLE, $\mathrm{B}_{\mathrm{SL}}, \mathrm{B}_{\mathrm{QL}}, \mathrm{EB}$, and $\mathrm{EB}_{\text {boot, with hyperparameters }}$ $a=2$ and $b=2$ for the Bayesian methods. For all magnitudes of sample sizes $n, \mathrm{~B}_{\mathrm{SL}}$ outperformed the others with the lowest MSE for true parameter $\theta=0.5,1$, or 2 while MLE was the best for $\theta=5,10$, or 20 . When focusing on the EB approaches, we found that the $\mathrm{EB}$ and $\mathrm{EB}_{\text {boot }}$ performances were very close to each other for all values of hyperparameters tested. Although the EB approaches did not provide the lowest MSEs, they were very close to those of the best estimator in each case. 
Table 2 summarizes the MSE values obtained by the five methods, with hyperparameter $a=2$ and $b=4$ for the Bayesian methods. For sample sizes $n=5,10,15$, or 20, the lowest MSE values were obtained by $\mathrm{B}_{\mathrm{SL}}$ for $\theta=0.5$ or 1 ; by $\mathrm{B}_{\mathrm{QL}}$ for $\theta=2$; by MLE for $\theta=5,10$, or 20 . For sample sizes $n=30,50$, or 100, the lowest MSE values were obtained by $\mathrm{B}_{\mathrm{SL}}$ for $\theta=0.5$; by $\mathrm{B}_{\mathrm{QL}}$ for $\theta=1$ or 2 ; and by MLE for $\theta=5,10$, or 20. Meanwhile, EB and EB boot $\mathrm{yielded}$ low MSEs close to the minimum MSE in each case.

The results in Table 3 comprise MSE values obtained by the five methods, with hyperparameters $a=1$ and $b=3.5$ for the Bayesian methods. This time, the lowest MSE values were obtained by $\mathrm{B}_{\mathrm{SL}}$ for $\theta=0.5$; by $\mathrm{B}_{\mathrm{QL}}$ for $\theta=1$ or 2 ;

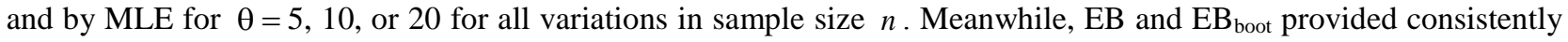
low MSEs that were only slightly higher than the others.

The MSE results in Table 4 for the five methods (with hyperparameters $a=4$ and $b=4$ for the Bayesian methods) indicate that for sample sizes $n=5$ or 10 , the lowest MSE values were obtained by $\mathrm{B}_{\mathrm{SL}}$ for $\theta=0.5$ or 1 ; by $\mathrm{B}_{\mathrm{QL}}$ for $\theta=$ 2; and by MLE for $\theta=5,10$, or 20. For sample sizes $n=15,20,30,50$, or 100, the lowest MSE values were obtained by $\mathrm{B}_{\mathrm{SL}}$ for $\theta=1$, by $\mathrm{B}_{\mathrm{QL}}$ for $\theta=2$, and by MLE for $\theta=0.5,5,10$, or 20 . Although $\mathrm{EB}$ and $\mathrm{EB}_{\text {boot }}$ performed worse than the others, their MSEs were close to the lowest MSE in each case.

Figure 2 exhibits the point estimation performances of the MLE, $\mathrm{B}_{\mathrm{SL}}, \mathrm{B}_{\mathrm{QL}}, \mathrm{EB}$, and $\mathrm{EB}_{\mathrm{boot}}$ methods in terms of MSE for various sample sizes. The results show that the MSE values of all methods increased with increasing $\theta$ but decreased with increasing sample size. All five point estimation methods provided similar MSE values for $\theta=0.5,1,2$, or 5 and all magnitudes of sample size. For $\theta=10$ and 20, the MSE values obtained with $\mathrm{B}_{\mathrm{SL}}$ exceeded the values obtained with the other methods, and especially for small sample sizes $(n=5,10,15,20$, or 30$)$, the MSE values from the $\mathrm{B}_{\mathrm{SL}}$ method increased dramatically. This means that the point estimation performance of $\mathrm{B}_{\mathrm{SL}}$ was weaker when parameter $\theta$ was large. On the other hand, when $\theta$ was small $(0.5,1$, or 2$), \mathrm{B}_{\mathrm{SL}}$ outperformed the other methods (Tables 1 to 4$)$.

Table 1. MSE of different estimators of Poisson distribution when $a=2, b=2$.

\begin{tabular}{|c|c|c|c|c|c|c|}
\hline \multirow{2}{*}{$n$} & \multirow{2}{*}{$\theta$} & \multicolumn{5}{|c|}{ Estimators } \\
\hline & & MLE & $\mathbf{B}_{\mathrm{SL}}$ & $\mathbf{B}_{\mathrm{QL}}$ & EB & $\mathbf{E B}_{\text {Boot }}$ \\
\hline \multirow{6}{*}{5} & 0.5 & 0.099104 & 0.091252 & 0.303076 & 0.113081 & 0.110512 \\
\hline & 1 & 0.201176 & 0.130994 & 0.420673 & 0.235998 & 0.232976 \\
\hline & 2 & 0.390496 & 0.326934 & 0.664979 & 0.461788 & 0.467555 \\
\hline & 5 & 1.02084 & 1.904548 & 1.389500 & 1.200347 & 1.211237 \\
\hline & 10 & 2.001792 & 7.893585 & 2.544969 & 2.381971 & 2.399838 \\
\hline & 20 & 4.090784 & 31.606460 & 5.110772 & 4.852462 & 4.836759 \\
\hline \multirow{6}{*}{10} & 0.5 & 0.049380 & 0.029017 & 0.045979 & 0.054428 & 0.053113 \\
\hline & 1 & 0.096838 & 0.075536 & 0.075940 & 0.106640 & 0.105970 \\
\hline & 2 & 0.196300 & 0.182111 & 0.258166 & 0.217606 & 0.214408 \\
\hline & 5 & 0.499204 & 0.824395 & 0.598437 & 0.545319 & 0.551117 \\
\hline & 10 & 0.989210 & 3.004691 & 1.147334 & 1.076708 & 1.089071 \\
\hline & 20 & 2.008994 & 11.615900 & 2.240245 & 2.220490 & 2.206249 \\
\hline \multirow{6}{*}{15} & 0.5 & 0.033924 & 0.031941 & 0.053973 & 0.035728 & 0.035843 \\
\hline & 1 & 0.068073 & 0.056555 & 0.090823 & 0.072484 & 0.072344 \\
\hline & 2 & 0.132362 & 0.125391 & 0.159198 & 0.140963 & 0.142610 \\
\hline & 5 & 0.349068 & 0.510380 & 0.385848 & 0.377020 & 0.366932 \\
\hline & 10 & 0.685924 & 1.646210 & 0.746185 & 0.736236 & 0.727646 \\
\hline & 20 & 0.976786 & 3.849628 & 1.030730 & 1.035781 & 1.029384 \\
\hline \multirow{6}{*}{20} & 0.5 & 0.024987 & 0.024233 & 0.037006 & 0.026291 & 0.025897 \\
\hline & 1 & 0.048071 & 0.041569 & 0.059630 & 0.050687 & 0.050784 \\
\hline & 2 & 0.099339 & 0.094697 & 0.114555 & 0.103102 & 0.104932 \\
\hline & 5 & 0.250262 & 0.345230 & 0.277183 & 0.261497 & 0.263243 \\
\hline & 10 & 0.486903 & 1.072216 & 0.524965 & 0.514545 & 0.513949 \\
\hline & 20 & 1.016642 & 3.917869 & 1.080546 & 1.059799 & 1.057904 \\
\hline \multirow{6}{*}{30} & 0.5 & 0.016293 & 0.015818 & 0.021430 & 0.016794 & 0.016898 \\
\hline & 1 & 0.033214 & 0.030335 & 0.038148 & 0.034263 & 0.034054 \\
\hline & 2 & 0.064005 & 0.062170 & 0.071148 & 0.066232 & 0.066094 \\
\hline & 5 & 0.163170 & 0.211318 & 0.174088 & 0.167863 & 0.168760 \\
\hline & 10 & 0.338277 & 0.613403 & 0.356621 & 0.350991 & 0.348555 \\
\hline & 20 & 0.646079 & 2.022373 & 0.673131 & 0.669031 & 0.665133 \\
\hline
\end{tabular}




\begin{tabular}{|c|c|c|c|c|c|c|}
\hline \multirow{6}{*}{50} & 0.5 & 0.009847 & 0.009557 & 0.011452 & 0.010040 & 0.009996 \\
\hline & 1 & 0.019904 & 0.018929 & 0.021741 & 0.020203 & 0.020323 \\
\hline & 2 & 0.039772 & 0.038868 & 0.041909 & 0.040349 & 0.041014 \\
\hline & 5 & 0.098478 & 0.114958 & 0.102239 & 0.100793 & 0.100468 \\
\hline & 10 & 0.201525 & 0.319802 & 0.206486 & 0.206050 & 0.205508 \\
\hline & 20 & 0.398703 & 0.909311 & 0.407968 & 0.408042 & 0.404783 \\
\hline \multirow{6}{*}{100} & 0.5 & 0.004860 & 0.004838 & 0.005317 & 0.004899 & 0.004906 \\
\hline & 1 & 0.010231 & 0.009943 & 0.010748 & 0.010319 & 0.010281 \\
\hline & 2 & 0.019386 & 0.019273 & 0.019927 & 0.019588 & 0.019606 \\
\hline & 5 & 0.051752 & 0.056191 & 0.052534 & 0.052242 & 0.052582 \\
\hline & 10 & 0.100112 & 0.128760 & 0.101749 & 0.101165 & 0.100730 \\
\hline & 20 & 0.198776 & 0.328405 & 0.201395 & 0.200816 & 0.201257 \\
\hline
\end{tabular}

Table 2. MSE of different estimators of Poisson distribution when $a=2, b=4$.

\begin{tabular}{|c|c|c|c|c|c|c|}
\hline \multirow{2}{*}{$n$} & \multirow{2}{*}{$\theta$} & \multicolumn{5}{|c|}{ Estimators } \\
\hline & & MLE & $\mathbf{B}_{\mathrm{SL}}$ & $\mathbf{B}_{\mathrm{QL}}$ & EB & EB $_{\text {Boot }}$ \\
\hline \multirow{6}{*}{5} & 0.5 & 0.193056 & 0.124405 & 0.130901 & 0.226791 & 0.234690 \\
\hline & 1 & 0.194896 & 0.125003 & 0.127759 & 0.225084 & 0.228800 \\
\hline & 2 & 0.389568 & 0.605250 & 0.330863 & 0.467007 & 0.454938 \\
\hline & 5 & 0.987616 & 4.432340 & 1.967464 & 1.192460 & 1.170282 \\
\hline & 10 & 2.016640 & 18.658890 & 7.903486 & 2.387870 & 2.448923 \\
\hline & 20 & 4.028128 & 76.554400 & 32.105830 & 4.814068 & 4.903727 \\
\hline \multirow{6}{*}{10} & 0.5 & 0.050622 & 0.029126 & 0.046228 & 0.054692 & 0.054099 \\
\hline & 1 & 0.096402 & 0.075086 & 0.075646 & 0.106676 & 0.105394 \\
\hline & 2 & 0.202824 & 0.299329 & 0.185010 & 0.223185 & 0.221703 \\
\hline & 5 & 0.501806 & 1.935992 & 0.822112 & 0.552956 & 0.550252 \\
\hline & 10 & 1.012412 & 8.024528 & 3.095003 & 1.120841 & 1.103653 \\
\hline & 20 & 1.987440 & 32.197900 & 11.526990 & 2.180303 & 2.188647 \\
\hline \multirow{6}{*}{15} & 0.5 & 0.033326 & 0.022549 & 0.031063 & 0.035105 & 0.035181 \\
\hline & 1 & 0.066933 & 0.056037 & 0.056983 & 0.069727 & 0.071655 \\
\hline & 2 & 0.136877 & 0.193247 & 0.128325 & 0.144046 & 0.144282 \\
\hline & 5 & 0.333276 & 1.089243 & 0.485680 & 0.354739 & 0.361134 \\
\hline & 10 & 0.669480 & 4.439124 & 1.679942 & 0.720296 & 0.714857 \\
\hline & 20 & 1.353934 & 17.746350 & 6.134013 & 1.447406 & 1.436745 \\
\hline \multirow{6}{*}{20} & 0.5 & 0.025197 & 0.018496 & 0.023837 & 0.026225 & 0.026087 \\
\hline & 1 & 0.049681 & 0.042573 & 0.043099 & 0.052011 & 0.051669 \\
\hline & 2 & 0.098797 & 0.134372 & 0.093235 & 0.103673 & 0.103373 \\
\hline & 5 & 0.251608 & 0.749857 & 0.352579 & 0.264320 & 0.264829 \\
\hline & 10 & 0.473187 & 2.865952 & 1.085616 & 0.492836 & 0.494520 \\
\hline & 20 & 1.011615 & 11.252580 & 3.821647 & 1.059451 & 1.054805 \\
\hline \multirow{6}{*}{30} & 0.5 & 0.016795 & 0.013526 & 0.016360 & 0.017247 & 0.017373 \\
\hline & 1 & 0.033546 & 0.030866 & 0.030633 & 0.034612 & 0.034714 \\
\hline & 2 & 0.032744 & 0.030045 & 0.029769 & 0.033704 & 0.033724 \\
\hline & 5 & 0.162496 & 0.416173 & 0.214092 & 0.167617 & 0.168261 \\
\hline & 10 & 0.330746 & 1.504826 & 0.610592 & 0.343417 & 0.341874 \\
\hline & 20 & 0.65508 & 5.765841 & 1.994743 & 0.677318 & 0.676764 \\
\hline \multirow{6}{*}{50} & 0.5 & 0.010090 & 0.008862 & 0.009862 & 0.010323 & 0.010201 \\
\hline & 1 & 0.020328 & 0.019206 & 0.019139 & 0.020668 & 0.020729 \\
\hline & 2 & 0.039910 & 0.047207 & 0.039120 & 0.040642 & 0.040870 \\
\hline & 5 & 0.100505 & 0.196560 & 0.117192 & 0.102418 & 0.102749 \\
\hline & 10 & 0.201708 & 0.672322 & 0.311498 & 0.204678 & 0.207057 \\
\hline & 20 & 0.404519 & 2.468836 & 0.931205 & 0.410182 & 0.412696 \\
\hline
\end{tabular}




\begin{tabular}{cccccccc}
\hline & 0.5 & 0.004917 & 0.004575 & 0.004850 & 0.004981 & 0.004976 \\
& 1 & 0.010109 & 0.009868 & 0.009833 & 0.010220 & 0.010209 \\
& 2 & 0.019633 & 0.021411 & 0.019333 & 0.019885 \\
& 5 & 0.049455 & 0.074940 & 0.053606 & 0.049950 & 0.049847 \\
& 10 & 0.100845 & 0.231952 & 0.131336 & 0.101993 \\
\hline
\end{tabular}

Table 3. MSE of different estimators of Poisson distribution when $a=1, b=3.5$.

\begin{tabular}{|c|c|c|c|c|c|c|}
\hline \multirow{2}{*}{$n$} & \multirow{2}{*}{$\theta$} & \multicolumn{5}{|c|}{ Estimators } \\
\hline & & MLE & $\mathbf{B}_{\mathrm{SL}}$ & $\mathbf{B}_{\mathrm{QL}}$ & EB & EB $_{\text {Boot }}$ \\
\hline \multirow{6}{*}{5} & 0.5 & 0.098816 & 0.050447 & 0.078236 & 0.113964 & 0.112924 \\
\hline & 1 & 0.208144 & 0.177792 & 0.159188 & 0.239794 & 0.246682 \\
\hline & 2 & 0.382824 & 0.649632 & 0.365832 & 0.450044 & 0.458498 \\
\hline & 5 & 0.995288 & 4.206471 & 1.730495 & 1.167655 & 1.168030 \\
\hline & 10 & 2.014328 & 16.810190 & 6.126311 & 2.383784 & 2.448520 \\
\hline & 20 & 4.103576 & 67.551330 & 22.761530 & 4.884260 & 4.838685 \\
\hline \multirow{6}{*}{10} & 0.5 & 0.050112 & 0.034032 & 0.043090 & 0.054152 & 0.053884 \\
\hline & 1 & 0.101258 & 0.093709 & 0.086043 & 0.110660 & 0.111894 \\
\hline & 2 & 0.205040 & 0.321132 & 0.199921 & 0.221530 & 0.224819 \\
\hline & 5 & 0.497482 & 1.823945 & 0.748054 & 0.547680 & 0.542045 \\
\hline & 10 & 0.998318 & 6.933701 & 2.320960 & 1.090330 & 1.087383 \\
\hline & 20 & 2.080266 & 27.346320 & 8.020482 & 2.277475 & 2.261644 \\
\hline \multirow{6}{*}{15} & 0.5 & 0.033710 & 0.025487 & 0.029654 & 0.035561 & 0.035911 \\
\hline & 1 & 0.064460 & 0.063921 & 0.058381 & 0.069243 & 0.068932 \\
\hline & 2 & 0.131875 & 0.195334 & 0.130171 & 0.138891 & 0.141515 \\
\hline & 5 & 0.332070 & 1.024969 & 0.446691 & 0.359863 & 0.355129 \\
\hline & 10 & 0.669570 & 3.886330 & 1.334571 & 0.717756 & 0.710855 \\
\hline & 20 & 1.324652 & 14.932590 & 4.288395 & 1.396016 & 1.418584 \\
\hline \multirow{6}{*}{20} & 0.5 & 0.024829 & 0.020238 & 0.022701 & 0.025765 & 0.025932 \\
\hline & 1 & 0.048869 & 0.048007 & 0.045057 & 0.050567 & 0.050542 \\
\hline & 2 & 0.098541 & 0.140529 & 0.098200 & 0.104324 & 0.103266 \\
\hline & 5 & 0.253787 & 0.673977 & 0.319856 & 0.264830 & 0.267035 \\
\hline & 10 & 0.501309 & 2.483548 & 0.878890 & 0.526486 & 0.521736 \\
\hline & 20 & 0.977366 & 9.331528 & 2.686589 & 1.031685 & 1.039903 \\
\hline \multirow{6}{*}{30} & 0.5 & 0.016378 & 0.014188 & 0.015409 & 0.016722 & 0.016807 \\
\hline & 1 & 0.032973 & 0.032750 & 0.031430 & 0.034075 & 0.034026 \\
\hline & 2 & 0.066745 & 0.087901 & 0.066593 & 0.069067 & 0.069040 \\
\hline & 5 & 0.166107 & 0.382418 & 0.200666 & 0.171428 & 0.171133 \\
\hline & 10 & 0.325179 & 1.291212 & 0.495207 & 0.339034 & 0.334482 \\
\hline & 20 & 0.664770 & 4.744609 & 1.447919 & 0.689368 & 0.688527 \\
\hline \multirow{6}{*}{50} & 0.5 & 0.010057 & 0.009258 & 0.009560 & 0.010235 & 0.010219 \\
\hline & 1 & 0.019962 & 0.020229 & 0.019227 & 0.020242 & 0.020416 \\
\hline & 2 & 0.039810 & 0.047227 & 0.039513 & 0.040570 & 0.040687 \\
\hline & 5 & 0.099396 & 0.179104 & 0.109629 & 0.101381 & 0.101704 \\
\hline & 10 & 0.197786 & 0.586977 & 0.268928 & 0.202314 & 0.202123 \\
\hline & 20 & 0.413135 & 2.029550 & 0.715810 & 0.419022 & 0.420516 \\
\hline \multirow{6}{*}{100} & 0.5 & 0.005020 & 0.004768 & 0.004930 & 0.005074 & 0.005073 \\
\hline & 1 & 0.009843 & 0.009873 & 0.009673 & 0.009872 & 0.009956 \\
\hline & 2 & 0.019963 & 0.022189 & 0.019815 & 0.020108 & 0.020250 \\
\hline & 5 & 0.051148 & 0.073086 & 0.054014 & 0.051684 & 0.051919 \\
\hline & 10 & 0.101070 & 0.204408 & 0.118711 & 0.101971 & 0.102117 \\
\hline & 20 & 0.205896 & 0.644675 & 0.285679 & 0.207335 & 0.207835 \\
\hline
\end{tabular}


Table 4. MSE of different estimators of Poisson distribution when $a=4, b=4$.

\begin{tabular}{|c|c|c|c|c|c|c|}
\hline \multirow{2}{*}{$n$} & \multirow{2}{*}{$\theta$} & \multicolumn{5}{|c|}{ Estimators } \\
\hline & & MLE & $\mathbf{B}_{\mathrm{SL}}$ & $\mathbf{B}_{\mathrm{QL}}$ & EB & $\mathbf{E B}_{\text {Boot }}$ \\
\hline \multirow{6}{*}{5} & 0.5 & 0.100128 & 0.098743 & 0.263371 & 0.114641 & 0.115051 \\
\hline & 1 & 0.195960 & 0.083822 & 0.222572 & 0.230449 & 0.231624 \\
\hline & 2 & 0.405232 & 0.356165 & 0.263037 & 0.478373 & 0.473320 \\
\hline & 5 & 1.010904 & 3.516952 & 1.350870 & 1.202746 & 1.207529 \\
\hline & 10 & 1.999648 & 16.702050 & 6.392148 & 2.419265 & 2.430623 \\
\hline & 20 & 3.969584 & 72.647800 & 28.971470 & 4.771550 & 4.831326 \\
\hline \multirow{6}{*}{10} & 0.5 & 0.051434 & 0.050878 & 0.102122 & 0.056908 & 0.055128 \\
\hline & 1 & 0.101432 & 0.059270 & 0.107722 & 0.110767 & 0.110743 \\
\hline & 2 & 0.198226 & 0.193303 & 0.153180 & 0.218184 & 0.217774 \\
\hline & 5 & 0.491562 & 1.581361 & 0.636411 & 0.534177 & 0.540410 \\
\hline & 10 & 0.974770 & 7.124140 & 2.490179 & 1.079565 & 1.077697 \\
\hline & 20 & 2.064076 & 31.090050 & 10.826180 & 2.290927 & 2.236347 \\
\hline \multirow{6}{*}{15} & 0.5 & 0.033396 & 0.033792 & 0.058953 & 0.035357 & 0.035628 \\
\hline & 1 & 0.066196 & 0.044897 & 0.070442 & 0.069794 & 0.070221 \\
\hline & 2 & 0.128922 & 0.134221 & 0.108821 & 0.136559 & 0.138378 \\
\hline & 5 & 0.333124 & 0.920332 & 0.394654 & 0.357071 & 0.353144 \\
\hline & 10 & 0.687593 & 3.964010 & 1.416239 & 0.729413 & 0.734068 \\
\hline & 20 & 1.350105 & 16.838740 & 5.567503 & 1.422659 & 1.428900 \\
\hline \multirow{6}{*}{20} & 0.5 & 0.024077 & 0.024649 & 0.039265 & 0.025165 & 0.025444 \\
\hline & 1 & 0.051149 & 0.037625 & 0.053279 & 0.054460 & 0.053690 \\
\hline & 2 & 0.101576 & 0.104136 & 0.088298 & 0.104857 & 0.107049 \\
\hline & 5 & 0.252716 & 0.628713 & 0.292682 & 0.265554 & 0.267322 \\
\hline & 10 & 0.506098 & 2.624467 & 0.968324 & 0.534303 & 0.534119 \\
\hline & 20 & 0.995604 & 10.862430 & 3.581061 & 1.050006 & 1.035090 \\
\hline \multirow{6}{*}{30} & 0.5 & 0.016563 & 0.016616 & 0.023824 & 0.017149 & 0.017237 \\
\hline & 1 & 0.034366 & 0.027512 & 0.034932 & 0.035673 & 0.035712 \\
\hline & 2 & 0.065286 & 0.067196 & 0.059680 & 0.067438 & 0.067591 \\
\hline & 5 & 0.167061 & 0.359689 & 0.188329 & 0.172232 & 0.171931 \\
\hline & 10 & 0.346848 & 1.398092 & 0.567188 & 0.356259 & 0.358324 \\
\hline & 20 & 0.681776 & 5.544798 & 1.885139 & 0.711597 & 0.702547 \\
\hline \multirow{6}{*}{50} & 0.5 & 0.009799 & 0.009962 & 0.012544 & 0.009994 & 0.009961 \\
\hline & 1 & 0.019790 & 0.017343 & 0.020198 & 0.020098 & 0.020137 \\
\hline & 2 & 0.040080 & 0.040739 & 0.037857 & 0.040819 & 0.041019 \\
\hline & 5 & 0.100763 & 0.175407 & 0.108698 & 0.103098 & 0.102907 \\
\hline & 10 & 0.198738 & 0.619418 & 0.282222 & 0.202806 & 0.204290 \\
\hline & 20 & 0.394630 & 2.298448 & 0.834769 & 0.403044 & 0.400599 \\
\hline \multirow{6}{*}{100} & 0.5 & 0.005159 & 0.005187 & 0.005922 & 0.005212 & 0.005225 \\
\hline & 1 & 0.010127 & 0.009450 & 0.010150 & 0.010248 & 0.010252 \\
\hline & 2 & 0.019272 & 0.019336 & 0.018720 & 0.019515 & 0.019455 \\
\hline & 5 & 0.050189 & 0.071657 & 0.052608 & 0.050808 & 0.050569 \\
\hline & 10 & 0.099895 & 0.214220 & 0.121308 & 0.100803 & 0.100747 \\
\hline & 20 & 0.196378 & 0.714174 & 0.314145 & 0.198759 & 0.198714 \\
\hline
\end{tabular}




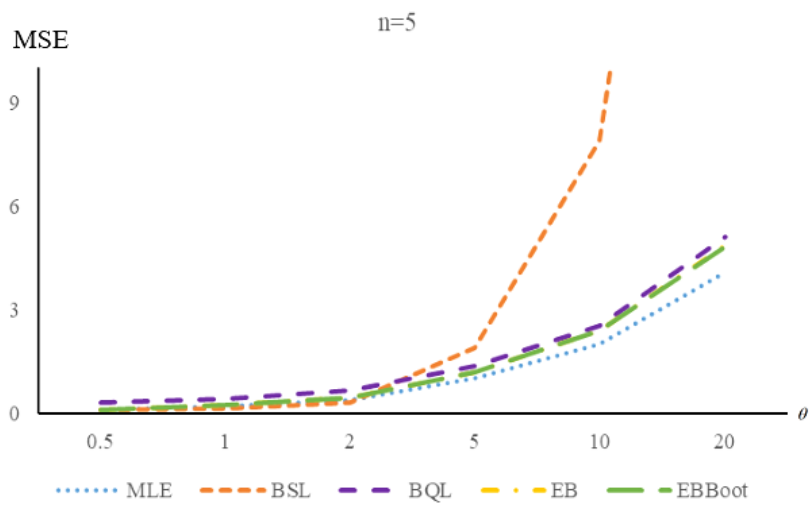

(a)

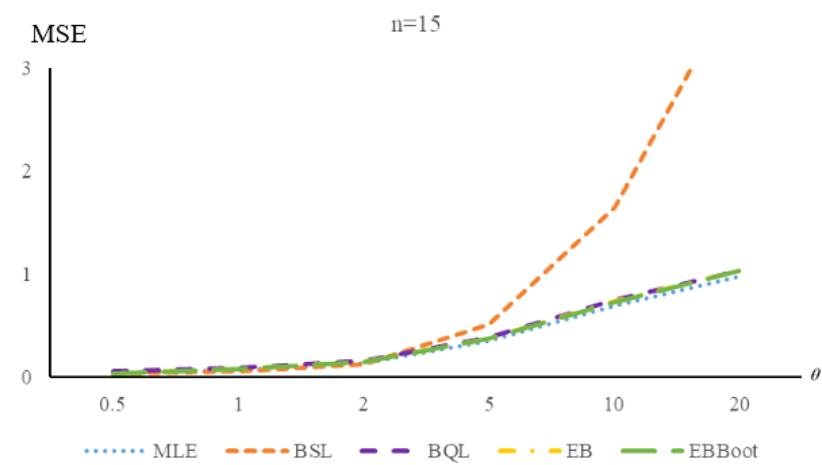

(c)

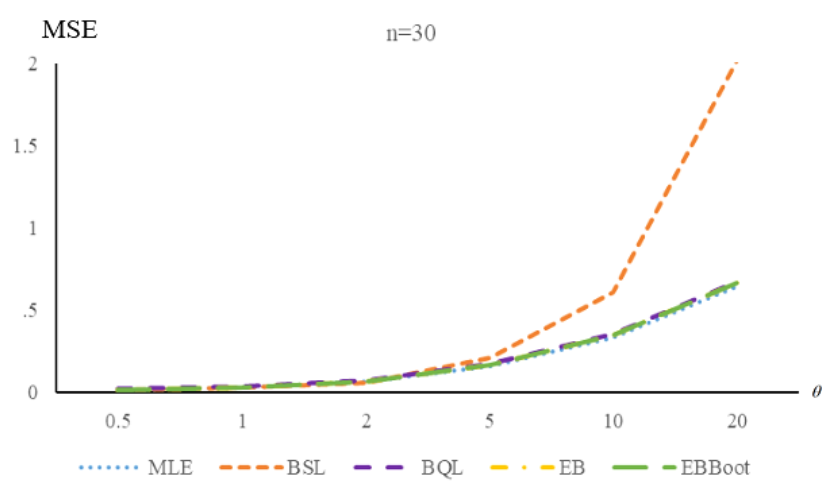

(e)

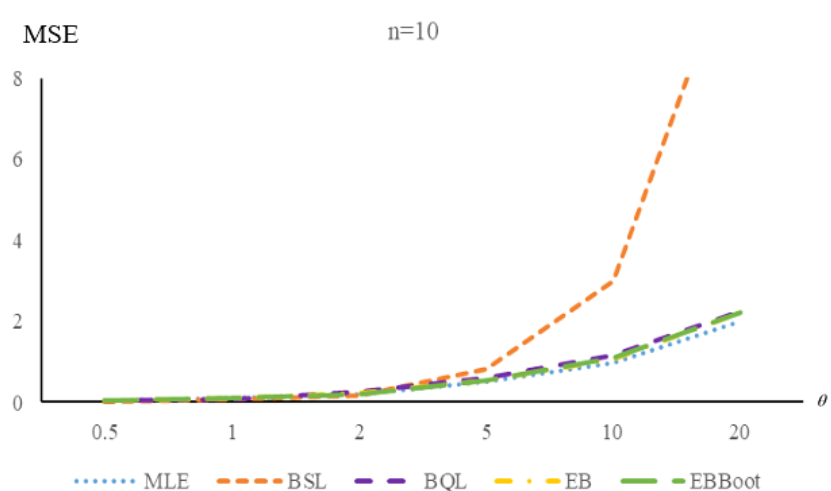

(b)

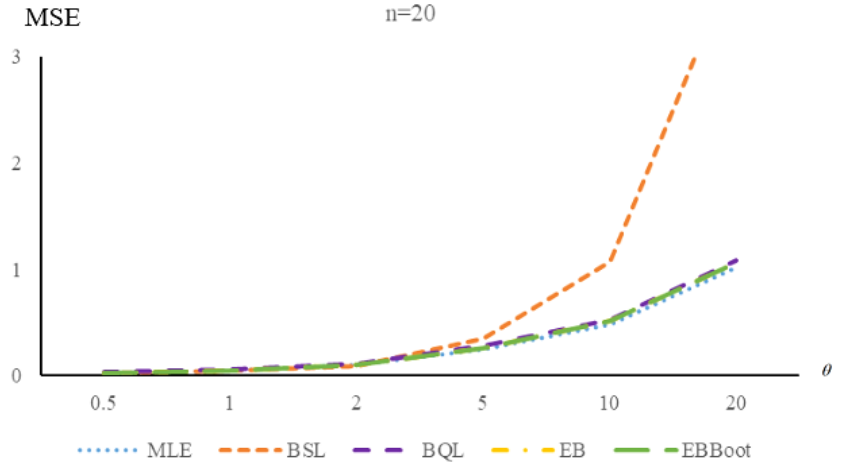

(d)

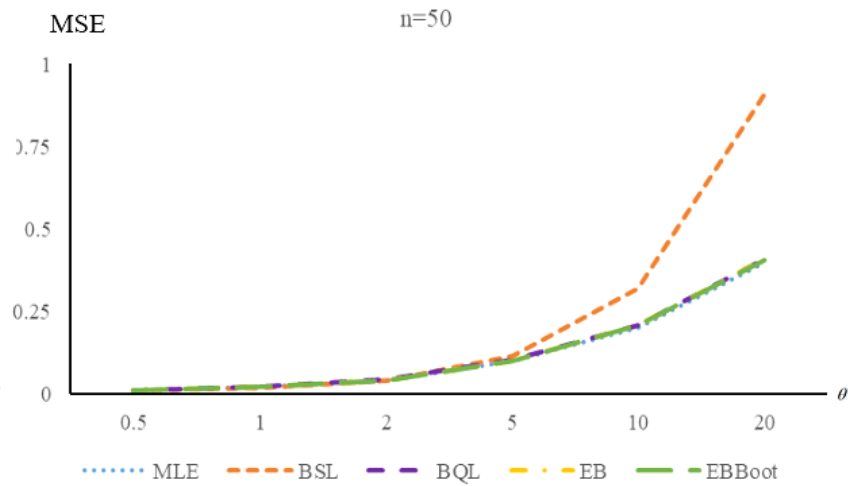

(f)

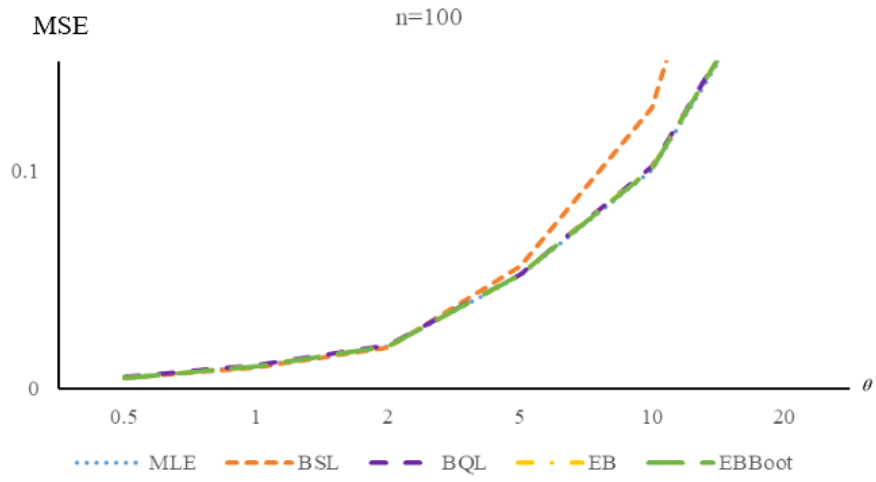

(g)

Figure 2. Comparative performance of point estimation. 


\section{3-2- Confident Interval}

The parameter settings for comparing the confidence interval performances were the same as for the point estimation simulation. Coverage probabilities (CPs) and average lengths (AL) were used to evaluate the performances of the methods. The most effective confidence interval method obtained a CP close to or greater than the nominal level of 0.95 and the shortest AL.

Table 5. Coverage probability (CP) and average length (AL) of $95 \%$ of confidence interval for the mean of Poisson distribution when $a=2, b=2$.

\begin{tabular}{|c|c|c|c|c|c|c|c|c|c|c|c|}
\hline \multirow{2}{*}{$n$} & \multirow{2}{*}{$\theta$} & \multicolumn{5}{|c|}{$\mathrm{CP}$} & \multicolumn{5}{|c|}{$\mathbf{A L}$} \\
\hline & & MLE & $\mathbf{B}_{\mathrm{SL}}$ & $\mathbf{B}_{\mathrm{QL}}$ & EB & $\mathbf{E B}_{\text {Boot }}$ & MLE & $\mathbf{B}_{\mathrm{SL}}$ & $\mathbf{B}_{\mathrm{QL}}$ & EB & $\mathbf{E B}_{\text {Boot }}$ \\
\hline \multirow{6}{*}{5} & 0.5 & 0.9106 & 0.6996 & 0.5494 & 0.5256 & 0.5386 & 1.607555 & 0.632610 & 0.890093 & 0.518620 & 0.517925 \\
\hline & 1 & 0.9580 & 0.6942 & 0.5926 & 0.5900 & 0.6024 & 2.412134 & 0.799737 & 1.120636 & 0.844432 & 0.844703 \\
\hline & 2 & 0.9706 & 0.5944 & 0.6180 & 0.6168 & 0.6116 & 3.470401 & 1.068726 & 1.482813 & 1.285845 & 1.288048 \\
\hline & 5 & 0.9864 & 0.3504 & 0.6340 & 0.6316 & 0.6316 & 5.520415 & 1.617572 & 2.270936 & 2.122267 & 2.128895 \\
\hline & 10 & 0.9926 & 0.1404 & 0.6366 & 0.6394 & 0.6340 & 7.834095 & 2.251841 & 3.126911 & 3.067146 & 3.056830 \\
\hline & 20 & 0.9932 & 0.0210 & 0.6456 & 0.6430 & 0.6380 & 11.08278 & 3.152468 & 4.439264 & 4.358594 & 4.311047 \\
\hline \multirow{6}{*}{10} & 0.5 & 0.9584 & 0.8258 & 0.7516 & 0.7192 & 0.7186 & 0.975306 & 0.603393 & 0.725451 & 0.542678 & 0.544237 \\
\hline & 1 & 0.9718 & 0.8138 & 0.7594 & 0.7568 & 0.7642 & 1.420385 & 0.810666 & 0.962627 & 0.835642 & 0.838655 \\
\hline & 2 & 0.9548 & 0.7528 & 0.7694 & 0.7804 & 0.7734 & 2.006209 & 1.094355 & 1.309834 & 1.225022 & 1.219264 \\
\hline & 5 & 0.9720 & 0.5920 & 0.7836 & 0.7760 & 0.7840 & 3.190490 & 1.712794 & 2.031309 & 1.965699 & 1.979353 \\
\hline & 10 & 0.9762 & 0.3774 & 0.7834 & 0.7866 & 0.7894 & 4.519046 & 2.383543 & 2.846706 & 2.814343 & 2.832078 \\
\hline & 20 & 0.9760 & 0.1518 & 0.8004 & 0.7820 & 0.7890 & 6.391771 & 3.350430 & 4.038289 & 3.975973 & 3.989187 \\
\hline \multirow{6}{*}{15} & 0.5 & 0.9404 & 0.8688 & 0.8206 & 0.8040 & 0.8002 & 0.769913 & 0.557150 & 0.631012 & 0.518728 & 0.516031 \\
\hline & 1 & 0.9578 & 0.8640 & 0.8376 & 0.8288 & 0.8326 & 1.097136 & 0.750590 & 0.851667 & 0.771445 & 0.772412 \\
\hline & 2 & 0.9542 & 0.8222 & 0.8352 & 0.8324 & 0.8400 & 1.559227 & 1.032362 & 1.167479 & 1.118811 & 1.116834 \\
\hline & 5 & 0.9668 & 0.7148 & 0.8386 & 0.8378 & 0.8442 & 2.470452 & 1.613843 & 1.834007 & 1.782541 & 1.800720 \\
\hline & 10 & 0.9718 & 0.5342 & 0.8434 & 0.8554 & 0.8436 & 3.499240 & 2.261647 & 2.570372 & 2.544284 & 2.550873 \\
\hline & 20 & 0.9614 & 0.2990 & 0.8430 & 0.8408 & 0.8394 & 4.952375 & 3.217632 & 3.638149 & 3.623931 & 3.608942 \\
\hline \multirow{6}{*}{20} & 0.5 & 0.9280 & 0.8904 & 0.8522 & 0.8408 & 0.8452 & 0.651502 & 0.510694 & 0.558601 & 0.482467 & 0.485399 \\
\hline & 1 & 0.9516 & 0.8798 & 0.8518 & 0.8510 & 0.8542 & 0.929861 & 0.697142 & 0.765758 & 0.710648 & 0.714416 \\
\hline & 2 & 0.9570 & 0.8562 & 0.8678 & 0.8592 & 0.8604 & 1.317382 & 0.964032 & 1.064626 & 1.023904 & 1.023173 \\
\hline & 5 & 0.9628 & 0.7694 & 0.8674 & 0.8704 & 0.8700 & 2.090040 & 1.512135 & 1.658402 & 1.637905 & 1.640942 \\
\hline & 10 & 0.9624 & 0.6430 & 0.8716 & 0.8706 & 0.8718 & 2.960116 & 2.131572 & 2.336404 & 2.320729 & 2.320040 \\
\hline & 20 & 0.9622 & 0.4158 & 0.8766 & 0.8746 & 0.8750 & 4.185683 & 3.007765 & 3.314869 & 3.301599 & 3.293631 \\
\hline \multirow{6}{*}{30} & 0.5 & 0.9224 & 0.9130 & 0.8858 & 0.8794 & 0.8848 & 0.501376 & 0.440308 & 0.472163 & 0.424875 & 0.426768 \\
\hline & 1 & 0.9278 & 0.8990 & 0.8860 & 0.8850 & 0.8834 & 0.714164 & 0.611624 & 0.650244 & 0.620488 & 0.618455 \\
\hline & 2 & 0.9522 & 0.8886 & 0.8910 & 0.8920 & 0.8928 & 1.009908 & 0.849029 & 0.910523 & 0.884960 & 0.884097 \\
\hline & 5 & 0.9520 & 0.8432 & 0.8986 & 0.8966 & 0.8926 & 1.600797 & 1.333246 & 1.427179 & 1.413425 & 1.412316 \\
\hline & 10 & 0.9516 & 0.7438 & 0.8972 & 0.8986 & 0.8974 & 2.262729 & 1.876996 & 2.004556 & 2.002416 & 2.002127 \\
\hline & 20 & 0.9510 & 0.5780 & 0.8938 & 0.8984 & 0.8918 & 3.200934 & 2.657712 & 2.837162 & 2.832057 & 2.828042 \\
\hline \multirow{6}{*}{50} & 0.5 & 0.9500 & 0.9286 & 0.9054 & 0.9042 & 0.9072 & 0.390226 & 0.358741 & 0.371205 & 0.351463 & 0.351061 \\
\hline & 1 & 0.9480 & 0.9188 & 0.9108 & 0.9082 & 0.9058 & 0.552758 & 0.497707 & 0.517736 & 0.502480 & 0.502273 \\
\hline & 2 & 0.9434 & 0.9088 & 0.9152 & 0.9090 & 0.9124 & 0.782867 & 0.700523 & 0.726801 & 0.717418 & 0.717098 \\
\hline & 5 & 0.9494 & 0.8764 & 0.9148 & 0.9152 & 0.9112 & 1.239173 & 1.098198 & 1.144472 & 1.138241 & 1.140552 \\
\hline & 10 & 0.9478 & 0.8218 & 0.9194 & 0.9176 & 0.9150 & 1.753233 & 1.552512 & 1.620128 & 1.605415 & 1.613848 \\
\hline & 20 & 0.9516 & 0.7204 & 0.9188 & 0.9218 & 0.9202 & 2.478789 & 2.199163 & 2.287030 & 2.279935 & 2.281450 \\
\hline \multirow{6}{*}{100} & 0.5 & 0.9450 & 0.9318 & 0.9216 & 0.9260 & 0.9210 & 0.276829 & 0.264330 & 0.269640 & 0.262297 & 0.261567 \\
\hline & 1 & 0.9456 & 0.9372 & 0.9312 & 0.9314 & 0.9318 & 0.391768 & 0.371713 & 0.377911 & 0.371974 & 0.373523 \\
\hline & 2 & 0.9464 & 0.9306 & 0.9346 & 0.9310 & 0.9302 & 0.554062 & 0.523320 & 0.532123 & 0.528149 & 0.529758 \\
\hline & 5 & 0.9428 & 0.9104 & 0.9258 & 0.9230 & 0.9268 & 0.876227 & 0.823006 & 0.840717 & 0.837201 & 0.839843 \\
\hline & 10 & 0.9462 & 0.8852 & 0.9276 & 0.9284 & 0.9288 & 1.239378 & 1.165336 & 1.187373 & 1.185568 & 1.188253 \\
\hline & 20 & 0.9514 & 0.8396 & 0.9370 & 0.9328 & 0.9356 & 1.753159 & 1.644476 & 1.682819 & 1.676053 & 1.679322 \\
\hline
\end{tabular}


Table 6. Coverage probability (CP) and average length (AL) of $95 \%$ of confidence interval for the mean of Poisson distribution when $a=2, b=4$.

\begin{tabular}{|c|c|c|c|c|c|c|c|c|c|c|c|}
\hline \multirow{2}{*}{$n$} & \multirow{2}{*}{$\theta$} & \multicolumn{5}{|c|}{ CP } & \multicolumn{5}{|c|}{$\mathbf{A L}$} \\
\hline & & MLE & $\mathbf{B}_{\mathrm{SL}}$ & $\mathbf{B}_{\mathrm{QL}}$ & EB & $\mathbf{E B}_{\text {Boot }}$ & MLE & $\mathbf{B}_{\mathrm{SL}}$ & $\mathbf{B}_{\mathrm{QL}}$ & EB & $\mathbf{E B}_{\text {Boot }}$ \\
\hline \multirow{6}{*}{5} & 0.5 & 0.9178 & 0.7138 & 0.6974 & 0.5454 & 0.5446 & 1.619054 & 0.489907 & 0.634784 & 0.522727 & 0.520517 \\
\hline & 1 & 0.9564 & 0.5538 & 0.6746 & 0.5746 & 0.5820 & 2.410230 & 0.625186 & 0.805963 & 0.844115 & 0.853402 \\
\hline & 2 & 0.9740 & 0.3116 & 0.5828 & 0.6052 & 0.6006 & 3.476643 & 0.829444 & 1.060156 & 1.282092 & 1.298908 \\
\hline & 5 & 0.9906 & 0.0486 & 0.3566 & 0.6258 & 0.6212 & 5.529801 & 1.263263 & 1.614621 & 2.129835 & 2.111677 \\
\hline & 10 & 0.9916 & 0.0010 & 0.1338 & 0.6306 & 0.6392 & 7.826954 & 1.743831 & 2.241875 & 2.986772 & 3.071715 \\
\hline & 20 & 0.9934 & 0.0000 & 0.0222 & 0.6526 & 0.6394 & 11.10013 & 2.47144 & 3.140614 & 4.387615 & 4.38398 \\
\hline \multirow{6}{*}{10} & 0.5 & 0.9634 & 0.8368 & 0.8278 & 0.7276 & 0.7406 & 0.982202 & 0.518259 & 0.605560 & 0.551392 & 0.551751 \\
\hline & 1 & 0.9658 & 0.7282 & 0.8142 & 0.7554 & 0.7510 & 1.411626 & 0.693180 & 0.801714 & 0.835429 & 0.837994 \\
\hline & 2 & 0.9540 & 0.5428 & 0.7572 & 0.7686 & 0.7690 & 2.011582 & .9402596 & 1.097768 & 1.223463 & 1.227203 \\
\hline & 5 & 0.9708 & 0.2010 & 0.5902 & 0.7762 & 0.7838 & 3.188316 & 1.451263 & 1.687773 & 1.970505 & 1.979217 \\
\hline & 10 & 0.9796 & 0.0328 & 0.392 & 0.7926 & 0.7888 & 4.522996 & 2.050157 & 2.389078 & 2.821249 & 2.816042 \\
\hline & 20 & 0.9774 & 0.0008 & 0.1644 & 0.7826 & 0.7896 & 6.39667 & 2.864764 & 3.350547 & 4.023185 & 4.011785 \\
\hline \multirow{6}{*}{15} & 0.5 & 0.9354 & 0.8650 & 0.8612 & 0.7990 & 0.7980 & 0.769246 & 0.498004 & 0.553855 & 0.520145 & 0.519903 \\
\hline & 1 & 0.9552 & 0.8088 & 0.8558 & 0.8230 & 0.8154 & 1.100694 & 0.673443 & 0.750170 & 0.771495 & 0.772001 \\
\hline & 2 & 0.9580 & 0.6596 & 0.8142 & 0.8372 & 0.8340 & 1.558429 & 0.927173 & 1.033403 & 1.119145 & 1.115776 \\
\hline & 5 & 0.9650 & 0.3498 & 0.7032 & 0.8348 & 0.8394 & 2.471100 & 1.440579 & 1.611227 & 1.784248 & 1.789175 \\
\hline & 10 & 0.9704 & 0.1042 & 0.5414 & 0.8442 & 0.8496 & 3.498840 & 2.028200 & 2.272177 & 2.553718 & 2.537857 \\
\hline & 20 & 0.9666 & 0.0070 & 0.3024 & 0.8366 & 0.8450 & 4.950921 & 2.862376 & 3.201113 & 3.597744 & 3.600471 \\
\hline \multirow{6}{*}{20} & 0.5 & 0.9330 & 0.8942 & 0.8854 & 0.8454 & 0.8440 & 0.653778 & 0.468232 & 0.511280 & 0.486629 & 0.484713 \\
\hline & 1 & 0.9572 & 0.8464 & 0.8830 & 0.8540 & 0.8614 & 0.928856 & 0.635397 & 0.693782 & 0.706837 & 0.712288 \\
\hline & 2 & 0.9588 & 0.7394 & 0.8518 & 0.8574 & 0.8662 & 1.321475 & 0.886235 & 0.968396 & 1.025802 & 1.020940 \\
\hline & 5 & 0.9598 & 0.4756 & 0.7702 & 0.8668 & 0.8702 & 2.091889 & 1.381536 & 1.508342 & 1.641463 & 1.637707 \\
\hline & 10 & 0.9600 & 0.2064 & 0.6406 & 0.8674 & 0.8626 & 2.960464 & 1.956247 & 2.136977 & 2.319926 & 2.319953 \\
\hline & 20 & 0.9634 & 0.0324 & 0.4286 & 0.8722 & 0.8682 & 4.186600 & 2.755330 & 3.010266 & 3.290685 & 3.300505 \\
\hline \multirow{6}{*}{30} & 0.5 & 0.9162 & 0.9120 & 0.9024 & 0.8806 & 0.8760 & 0.501499 & 0.414360 & 0.440025 & 0.427111 & 0.425545 \\
\hline & 1 & 0.9318 & 0.8838 & 0.9016 & 0.8880 & 0.8876 & 0.715524 & 0.574723 & 0.611604 & 0.620615 & 0.621832 \\
\hline & 2 & 0.9496 & 0.8134 & 0.8914 & 0.8946 & 0.8948 & 1.010807 & 0.799951 & 0.849725 & 0.884262 & 0.883668 \\
\hline & 5 & 0.9484 & 0.5966 & 0.8264 & 0.8844 & 0.8904 & 1.597668 & 1.255698 & 1.329586 & 1.405938 & 1.402207 \\
\hline & 10 & 0.9538 & 0.3632 & 0.7470 & 0.9010 & 0.8958 & 2.264022 & 1.769438 & 1.891281 & 1.987458 & 1.994811 \\
\hline & 20 & 0.9478 & 0.0976 & 0.5488 & 0.8986 & 0.8990 & 3.198483 & 2.506965 & 2.653921 & 2.825603 & 2.817794 \\
\hline \multirow{6}{*}{50} & 0.5 & 0.9502 & 0.9240 & 0.9248 & 62 & 0.9 & 0.390971 & 0.345088 & 0.359276 & 0.351181 & 0.351694 \\
\hline & 1 & 0.9440 & 0.8980 & 0.9102 & 0.9076 & 0.9066 & 0.552646 & 0.480353 & 0.497871 & 0.502346 & 0.503862 \\
\hline & 2 & 0.9426 & 0.8566 & 0.9054 & 0.9116 & 0.9030 & 0.783156 & 0.672858 & 0.699409 & 0.718351 & 0.714519 \\
\hline & 5 & 0.9462 & 0.7518 & 0.8844 & 0.9114 & 0.9102 & 1.240324 & 1.062735 & 1.103290 & 1.138516 & 1.138908 \\
\hline & 10 & 0.9488 & 0.5432 & 0.8192 & 0.9136 & 0.9182 & 1.751861 & 1.499732 & 1.557404 & 1.611256 & 1.611675 \\
\hline & 20 & 0.9472 & 0.2632 & 0.7142 & 0.9176 & 0.9100 & 2.478177 & 2.108174 & 2.195631 & 2.282742 & 2.277332 \\
\hline \multirow{6}{*}{100} & 0.5 & 0.9460 & 0.9350 & 0.9334 & 0.9318 & 0.9304 & 0.276062 & 0.258713 & 0.263430 & 0.261331 & 0.261406 \\
\hline & 1 & 0.9426 & 0.9264 & 0.9340 & 0.9308 & 0.9252 & 0.391698 & 0.363462 & 0.371062 & 0.373335 & 0.372599 \\
\hline & 2 & 0.9482 & 0.9086 & 0.934 & 0.9324 & 0.9330 & 0.554335 & 0.513291 & 0.523896 & 0.530182 & 0.528249 \\
\hline & 5 & 0.9504 & 0.8448 & 0.9106 & 0.9296 & 0.9300 & 0.876142 & 0.808593 & 0.823742 & 0.837359 & 0.838808 \\
\hline & 10 & 0.9508 & 0.7386 & 0.8890 & 0.9342 & 0.9332 & 1.238915 & 1.143413 & 1.166334 & 1.189681 & 1.186145 \\
\hline & 20 & 0.9480 & 0.5594 & 0.8332 & 0.9280 & 0.9278 & 1.753316 & 1.613821 & 1.646551 & 1.678923 & 1.678422 \\
\hline
\end{tabular}


Table 7. Coverage probability (CP) and average length (AL) of $95 \%$ of confidence interval for the mean of Poisson distribution when $a=1, b=3.5$.

\begin{tabular}{|c|c|c|c|c|c|c|c|c|c|c|c|}
\hline \multirow{2}{*}{$n$} & \multirow{2}{*}{$\theta$} & \multicolumn{5}{|c|}{$\mathbf{C P}$} & \multicolumn{5}{|c|}{$\mathbf{A L}$} \\
\hline & & MLE & $\mathbf{B}_{\mathrm{SL}}$ & $\mathbf{B}_{\mathrm{QL}}$ & EB & $\mathbf{E B}_{\text {Boot }}$ & MLE & $\mathbf{B}_{\mathrm{SL}}$ & $\mathbf{B}_{\mathrm{QL}}$ & EB & $\mathbf{E B}_{\text {Boot }}$ \\
\hline \multirow{6}{*}{5} & 0.5 & 0.9180 & 0.6172 & 0.6696 & 0.5410 & 0.5392 & 1.630927 & 0.452135 & 0.591007 & 0.535666 & 0.528802 \\
\hline & 1 & 0.9592 & 0.4812 & 0.6342 & 0.5858 & 0.5888 & 2.404796 & 0.607332 & 0.789203 & 0.837424 & 0.840202 \\
\hline & 2 & 0.9698 & 0.2962 & 0.5728 & 0.6110 & 0.6150 & 3.466534 & 0.845365 & 1.090610 & 1.289298 & 1.290306 \\
\hline & 5 & 0.9872 & 0.0660 & 0.4132 & 0.6356 & 0.6340 & 5.524026 & 1.300733 & 1.703790 & 2.120537 & 2.111179 \\
\hline & 10 & 0.9908 & 0.0064 & 0.2508 & 0.6296 & 0.6436 & 7.833084 & 1.838392 & 2.394342 & 3.049160 & 3.064594 \\
\hline & 20 & 0.9934 & 0.0000 & 0.0796 & 0.6494 & 0.6384 & 11.09148 & 2.598600 & 3.393944 & 4.369718 & 4.344879 \\
\hline \multirow{6}{*}{10} & 0.5 & 0.9594 & 0.7684 & 0.8036 & 0.7304 & 0.7282 & 0.979645 & 0.495225 & 0.579723 & 0.544429 & 0.545553 \\
\hline & 1 & 0.9692 & 0.6810 & 0.7916 & 0.7606 & 0.7550 & 1.409392 & 0.680688 & 0.805592 & 0.837319 & 0.831482 \\
\hline & 2 & 0.9478 & 0.5188 & 0.7296 & 0.7614 & 0.7636 & 2.000704 & 0.949720 & 1.107310 & 1.212492 & 1.215983 \\
\hline & 5 & 0.9750 & 0.2434 & 0.6500 & 0.7908 & 0.7882 & 3.190506 & 1.498681 & 1.755422 & 1.984182 & 1.965789 \\
\hline & 10 & 0.9732 & 0.0638 & 0.5130 & 0.7746 & 0.7790 & 4.516912 & 2.104345 & 2.482233 & 2.814242 & 2.818787 \\
\hline & 20 & 0.9740 & 0.0040 & 0.3014 & 0.7850 & 0.7864 & 6.393036 & 2.985140 & 3.500916 & 3.982631 & 3.981626 \\
\hline \multirow{6}{*}{15} & 0.5 & 0.9376 & 0.8304 & 0.8604 & 0.7994 & 0.8016 & 0.767799 & 0.478725 & 0.540265 & 0.519082 & 0.516549 \\
\hline & 1 & 0.9556 & 0.7654 & 0.8382 & 0.8186 & 0.8150 & 1.095613 & 0.664168 & 0.746610 & 0.771155 & 0.768141 \\
\hline & 2 & 0.9586 & 0.6616 & 0.8166 & 0.8278 & 0.8286 & 1.561913 & 0.932263 & 1.056257 & 1.117456 & 1.115744 \\
\hline & 5 & 0.9690 & 0.3968 & 0.7532 & 0.8462 & 0.8490 & 2.472578 & 1.472772 & 1.644812 & 1.791164 & 1.791246 \\
\hline & 10 & 0.9736 & 0.1554 & 0.6552 & 0.8520 & 0.8564 & 3.499628 & 2.072363 & 2.329111 & 2.544133 & 2.554489 \\
\hline & 20 & 0.9644 & 0.0206 & 0.4546 & 0.8392 & 0.8348 & 4.949642 & 2.925940 & 3.292354 & 3.614396 & 3.598564 \\
\hline \multirow{6}{*}{20} & 0.5 & 0.9292 & 0.8600 & 0.8792 & 0.8428 & 0.8462 & 0.651479 & 0.452906 & 0.498307 & 0.482959 & 0.484167 \\
\hline & 1 & 0.9568 & 0.8298 & 0.8736 & 0.8626 & 0.8564 & 0.931360 & 0.638110 & 0.697209 & 0.714872 & 0.711225 \\
\hline & 2 & 0.9588 & 0.7342 & 0.8572 & 0.8666 & 0.8636 & 1.319243 & 0.896132 & 0.977165 & 1.028626 & 1.029791 \\
\hline & 5 & 0.9632 & 0.5130 & 0.7990 & 0.8666 & 0.8690 & 2.091244 & 1.405291 & 1.539872 & 1.642670 & 1.643886 \\
\hline & 10 & 0.9612 & 0.2578 & 0.7218 & 0.8740 & 0.8724 & 2.957603 & 1.985019 & 2.182842 & 2.318158 & 2.319276 \\
\hline & 20 & 0.9648 & 0.0660 & 0.5724 & 0.8722 & 0.8708 & 4.185874 & 2.809849 & 3.064425 & 3.297706 & 3.294691 \\
\hline \multirow{6}{*}{30} & 0.5 & 0.9126 & 0.8838 & 0.8976 & 0.8672 & 0.8714 & 0.5015533 & 0.4084347 & 0.4360317 & 0.428012 & 0.427603 \\
\hline & 1 & 0.9292 & 0.8634 & 0.8918 & 0.8872 & 0.8854 & 0.711836 & 0.5705448 & 0.6079093 & 0.612869 & 0.620041 \\
\hline & 2 & 0.9544 & 0.8020 & 0.8872 & 0.8984 & 0.8960 & 1.010261 & 0.807160 & 0.857172 & 0.885760 & 0.883386 \\
\hline & 5 & 0.9524 & 0.6492 & 0.8574 & 0.8952 & 0.8986 & 1.598787 & 1.270823 & 1.345487 & 1.407942 & 1.407048 \\
\hline & 10 & 0.9532 & 0.4294 & 0.7966 & 0.8976 & 0.8954 & 2.261131 & 1.799713 & 1.904499 & 1.996044 & 1.992668 \\
\hline & 20 & 0.9500 & 0.1778 & 0.6940 & 0.8932 & 0.8928 & 3.200161 & 2.538920 & 2.710243 & 2.827263 & 2.826159 \\
\hline \multirow{6}{*}{50} & 0.5 & 0.9506 & 0.9126 & 0.9136 & 0.9016 & 0.9036 & 0.390541 & 0.341258 & 0.354475 & 0.351300 & 0.352386 \\
\hline & 1 & 0.9438 & 0.8934 & 0.9110 & 0.9082 & 0.9084 & 0.553265 & 0.479373 & 0.498875 & 0.503006 & 0.502921 \\
\hline & 2 & 0.9502 & 0.8658 & 0.9122 & 0.9112 & 0.9152 & 0.783166 & 0.678508 & 0.702246 & 0.715435 & 0.718075 \\
\hline & 5 & 0.9534 & 0.7658 & 0.8864 & 0.9150 & 0.9160 & 1.239135 & 1.067899 & 1.112000 & 1.137311 & 1.137578 \\
\hline & 10 & 0.9432 & 0.6110 & 0.8532 & 0.9088 & 0.9076 & 1.752746 & 1.511201 & 1.568788 & 1.609130 & 1.611529 \\
\hline & 20 & 0.9498 & 0.3630 & 0.7864 & 0.9128 & 0.9182 & 2.477934 & 2.131182 & 2.213501 & 2.275756 & 2.280907 \\
\hline \multirow{6}{*}{100} & 0.5 & 0.9508 & 0.9314 & 0.9374 & 0.9310 & 0.9280 & 0.276173 & 0.257769 & 0.262750 & 0.261717 & 0.261530 \\
\hline & 1 & 0.9410 & 0.9162 & 0.9276 & 0.9286 & 0.9272 & 0.391393 & 0.363142 & 0.370356 & 0.372511 & 0.372505 \\
\hline & 2 & 0.9444 & 0.9066 & 0.9262 & 0.9330 & 0.9282 & 0.554361 & 0.514372 & 0.524701 & 0.528961 & 0.529943 \\
\hline & 5 & 0.9478 & 0.8650 & 0.9226 & 0.9330 & 0.9336 & 0.876456 & 0.811610 & 0.830240 & 0.839697 & 0.839246 \\
\hline & 10 & 0.9490 & 0.7756 & 0.9010 & 0.9316 & 0.9318 & 1.238900 & 1.146744 & 1.170690 & 1.187179 & 1.186867 \\
\hline & 20 & 0.9498 & 0.6302 & 0.8684 & 0.9324 & 0.9328 & 1.753073 & 1.622126 & 1.656973 & 1.679569 & 1.679983 \\
\hline
\end{tabular}


Table 8. Coverage probability (CP) and average length (AL) of $95 \%$ of confidence interval for the mean of Poisson distribution when $a=4, b=4$.

\begin{tabular}{|c|c|c|c|c|c|c|c|c|c|c|c|}
\hline \multirow{2}{*}{$n$} & \multirow{2}{*}{$\theta$} & \multicolumn{5}{|c|}{ CP } & \multicolumn{5}{|c|}{$\mathbf{A L}$} \\
\hline & & MLE & $\mathbf{B}_{\mathrm{SL}}$ & $\mathbf{B}_{\mathrm{QL}}$ & EB & $\mathbf{E B}_{\text {Boot }}$ & MLE & $\mathbf{B}_{\mathrm{SL}}$ & $\mathbf{B}_{\mathrm{QL}}$ & EB & $\mathbf{E B}_{\text {Boot }}$ \\
\hline \multirow{6}{*}{5} & 0.5 & 0.9138 & 0.6438 & 0.4156 & 0.5366 & 0.5386 & 1.624976 & 0.612501 & 0.779173 & 0.525449 & 0.527094 \\
\hline & 1 & 0.9616 & 0.7268 & 0.6554 & 0.5962 & 0.5832 & 2.406193 & 0.717967 & 0.916662 & 0.843736 & 0.856087 \\
\hline & 2 & 0.9682 & 0.4842 & 0.6736 & 0.6010 & 0.6080 & 3.465726 & 0.898523 & 1.149336 & 1.281356 & 1.275973 \\
\hline & 5 & 0.9872 & 0.0780 & 0.4468 & 0.6328 & 0.6218 & 5.510437 & 1.304584 & 1.653120 & 2.119507 & 2.111643 \\
\hline & 10 & 0.9906 & 0.0038 & 0.1920 & 0.6356 & 0.6282 & 7.834848 & 1.780249 & 2.307838 & 3.055042 & 3.058725 \\
\hline & 20 & 0.9930 & 0.0000 & 0.0294 & 0.6490 & 0.6602 & 11.092860 & 2.473280 & 3.196755 & 4.354572 & 4.375683 \\
\hline \multirow{6}{*}{10} & 0.5 & 0.9588 & 0.7976 & 0.6674 & 0.7286 & 0.7286 & 0.985056 & 0.600991 & 0.700818 & 0.550822 & 0.545220 \\
\hline & 1 & 0.9682 & 0.8480 & 0.8000 & 0.7736 & 0.7668 & 1.410883 & 0.748984 & 0.872630 & 0.834705 & 0.828385 \\
\hline & 2 & 0.9560 & 0.6948 & 0.8254 & 0.7852 & 0.7780 & 2.009617 & 0.983333 & 1.141522 & 1.224653 & 1.218208 \\
\hline & 5 & 0.9684 & 0.2798 & 0.6826 & 0.7822 & 0.7790 & 3.193513 & 1.485009 & 1.734832 & 1.975854 & 1.973136 \\
\hline & 10 & 0.9764 & 0.0414 & 0.4626 & 0.7892 & 0.7830 & 4.519447 & 2.058062 & 2.404176 & 2.814477 & 2.804773 \\
\hline & 20 & 0.9754 & 0.0014 & 0.1836 & 0.7990 & 0.7950 & 6.396466 & 2.903937 & 3.376389 & 4.018442 & 4.008764 \\
\hline \multirow{6}{*}{15} & 0.5 & 0.9338 & 0.8474 & 0.7600 & 0.8014 & 0.7988 & 0.766632 & 0.548009 & 0.613780 & 0.519500 & 0.515156 \\
\hline & 1 & 0.9584 & 0.8754 & 0.8460 & 0.8258 & 0.8202 & 1.099199 & 0.711726 & 0.793186 & 0.770361 & 0.770540 \\
\hline & 2 & 0.9608 & 0.7806 & 0.8694 & 0.8356 & 0.8380 & 1.560337 & 0.957583 & 1.073157 & 1.113441 & 1.117606 \\
\hline & 5 & 0.9676 & 0.4314 & 0.7786 & 0.8392 & 0.8362 & 2.474110 & 1.459141 & 1.637839 & 1.794852 & 1.781565 \\
\hline & 10 & 0.9676 & 0.1454 & 0.6022 & 0.8412 & 0.8406 & 3.501964 & 2.037875 & 2.278511 & 2.536040 & 2.542311 \\
\hline & 20 & 0.9672 & 0.0094 & 0.3290 & 0.8492 & 0.8492 & 4.950872 & 2.867101 & 3.212928 & 3.611731 & 3.607749 \\
\hline \multirow{6}{*}{20} & 0.5 & 0.9304 & 0.8696 & 0.8086 & 0.8410 & 0.8386 & 0.656562 & 0.506474 & 0.556824 & 0.488674 & 0.487875 \\
\hline & 1 & 0.9528 & 0.8942 & 0.8780 & 0.8574 & 0.8570 & 0.931435 & 0.668763 & 0.728887 & 0.713966 & 0.709739 \\
\hline & 2 & 0.9580 & 0.8148 & 0.8878 & 0.8678 & 0.8622 & 1.317849 & 0.904041 & 0.988854 & 1.020844 & 1.026129 \\
\hline & 5 & 0.9568 & 0.5234 & 0.8044 & 0.8650 & 0.8648 & 2.087496 & 1.392933 & 1.518305 & 1.634716 & 1.636990 \\
\hline & 10 & 0.9618 & 0.2318 & 0.6834 & 0.8722 & 0.8658 & 2.958177 & 1.965138 & 2.146741 & 2.322557 & 2.319068 \\
\hline & 20 & 0.9590 & 0.0348 & 0.452 & 0.8684 & 0.8722 & 4.184803 & 2.753412 & 3.014108 & 3.295433 & 3.298369 \\
\hline \multirow{6}{*}{30} & 0.5 & 0.9148 & 0.9022 & 0.8582 & 0.8742 & 0.8802 & 0.502206 & 0.440640 & 0.468760 & 0.426775 & 0.427025 \\
\hline & 1 & 0.9348 & 0.9166 & 0.9002 & 0.8932 & 0.8934 & 0.712650 & 0.593461 & 0.628450 & 0.617534 & 0.618670 \\
\hline & 2 & 0.9488 & 0.8602 & 0.9058 & 0.8906 & 0.8872 & 1.010039 & 0.812401 & 0.862668 & 0.885048 & 0.884317 \\
\hline & 5 & 0.9520 & 0.6728 & 0.8616 & 0.8982 & 0.8928 & 1.599982 & 1.264963 & 1.346935 & 1.411770 & 1.405024 \\
\hline & 10 & 0.9478 & 0.3938 & 0.7740 & 0.8926 & 0.8932 & 2.261469 & 1.783701 & 1.888889 & 1.992391 & 1.989169 \\
\hline & 20 & 0.9492 & 0.1052 & 0.6054 & 0.8964 & 0.9018 & 3.199392 & 2.497719 & 2.657876 & 2.828264 & 2.832740 \\
\hline \multirow{6}{*}{50} & 0.5 & 0.9494 & 0.9190 & 0.8958 & 0.9054 & 0.9008 & 0.389288 & 0.357244 & 0.369245 & 0.349355 & 0.349183 \\
\hline & 1 & 0.9512 & 0.9272 & 0.9194 & 0.9138 & 0.9126 & 0.552617 & 0.489920 & 0.507157 & 0.502239 & 0.502421 \\
\hline & 2 & 0.9434 & 0.8952 & 0.9182 & 0.9124 & 0.9046 & 0.783597 & 0.682145 & 0.706176 & 0.716969 & 0.715345 \\
\hline & 5 & 0.9422 & 0.7752 & 0.8906 & 0.9070 & 0.9070 & 1.238952 & 1.064611 & 1.105489 & 1.139260 & 1.136898 \\
\hline & 10 & 0.9484 & 0.5724 & 0.8468 & 0.9138 & 0.9144 & 1.751751 & 1.500611 & 1.556808 & 1.608410 & 1.611586 \\
\hline & 20 & 0.9520 & 0.2964 & 0.7364 & 0.9146 & 0.9206 & 2.479200 & 2.109114 & 2.191986 & 2.273197 & 2.280550 \\
\hline \multirow{6}{*}{100} & 0.5 & 0.9514 & 0.9366 & 0.9226 & 0.9298 & 0.9324 & 0.277015 & 0.264949 & 0.269998 & 0.262163 & 0.262541 \\
\hline & 1 & 0.9470 & 0.9336 & 0.9342 & 0.9366 & 0.9336 & 0.391244 & 0.366662 & 0.375084 & 0.372798 & 0.372697 \\
\hline & 2 & 0.9480 & 0.9246 & 0.9340 & 0.9346 & 0.9332 & 0.554255 & 0.516222 & 0.525529 & 0.529987 & 0.531118 \\
\hline & 5 & 0.9466 & 0.8776 & 0.9216 & 0.9352 & 0.9282 & 0.876653 & 0.812158 & 0.824949 & 0.840319 & 0.837829 \\
\hline & 10 & 0.9500 & 0.7566 & 0.8926 & 0.9362 & 0.9328 & 1.238742 & 1.141032 & 1.166202 & 1.186886 & 1.187039 \\
\hline & 20 & 0.9524 & 0.5736 & 0.8500 & 0.9348 & 0.9334 & 1.753047 & 1.616746 & 1.647908 & 1.680374 & 1.68149 \\
\hline
\end{tabular}

Tables 5 to 8 report the CPs and ALs obtained by the MLE, $\mathrm{B}_{\mathrm{SL}}, \mathrm{B}_{\mathrm{QL}}, \mathrm{EB}$, and $\mathrm{EB}_{\text {boot }}$ methods, with hyperparameters $(2,2),(2,4),(1,3.5)$, and $(4,4)$ for the Bayesian methods, respectively. The results show that for sample sizes $n=5,10$, 15,20 , or 30, the MLE method provided CP values close to 0.95 for all values of $\theta$, although its ALs were not the shortest. The results for small sample sizes indicate that when $\theta$ increased, the performances of $\mathrm{B}_{\mathrm{SL}}$ and $\mathrm{B}_{\mathrm{QL}}$ decreased 
with $\mathrm{CP}$ values lower than the nominal level of 0.95 .Although the CPs of EB and EB boot were not close to 0.95 , they improved with increasing of $\theta$. In addition, their AL values were similar to each other and narrower than the other methods for $\theta=0.5$.

For sample sizes $n=50$ or 100 , MLE once again obtained CP values close to 0.95 for all values of $\theta$, as did $\mathrm{B}_{\mathrm{SL}}$ and $\mathrm{B}_{\mathrm{QL}}$ for $\theta=0.5,1$, or 2 . Meanwhile, the $\mathrm{CPs}$ of $\mathrm{B}_{\mathrm{SL}}$ decreased drastically for large values of $\theta$ while those of $\mathrm{EB}$ and $\mathrm{EB}_{\mathrm{boot}}$ were similar to each other and higher than $\mathrm{B}_{\mathrm{SL}}$ and $\mathrm{B}_{\mathrm{QL}}$. Although the ALs obtained by the five methods were similar, slightly shorter ones were provided by $\mathrm{EB}_{\text {boot }}$ for $\theta=0.5$ and $\mathrm{B}_{\mathrm{SL}}$ for $\theta=1,2,5,10$, or 20 in the case of sample size $n=$ 50. Meanwhile, the shortest ALs were provided by $\mathrm{EB}$ for $\theta=0.5$ and $\mathrm{B}_{\mathrm{SL}}$ for $\theta=1,2,5$, 10, or 20 in the case of sample size $n=100$.

\section{4- Discussion}

Frequentist and Bayesian inference are fundamentally different principles in statistics. Estimating the Poisson parameter using the MLE method as a classical frequentist approach was proposed by Araveeporn [1] and Hassan et al. [2]. The existing EB and EB with bootstrapping approaches were derived by Supharakonsakun and Jampachasri [27]. Empirical Bayes methods are different from the classical Bayesian approach in that the hyperparameters are assumed to be unknown and prior observations are used to estimate them via the classical Bayesian approach. Determining the hyperparameters is important for the posterior distribution and increases the accuracy of the parameter estimation. Two different loss functions, squared-error and quadratic [12], for parameter estimation based on the classical Bayesian approach were used in this study.

The results in this study were similar to those of Srivastava [7] in that the Bayesian point estimation method under the squared-error loss function provided estimates nearer to the true value of the Poisson parameter. Besides, the Bayesian estimators under different loss functions performed better than the classical estimator (MLE) in the case of small and different values of the hyperparameters, which is similar to the findings of Hassan and Baizid [12] and Naji and Rasheed who found that Bayesian estimate parameters under precautionary [15], generalized weighted [14] or entropy [16] loss functions were the better than the classical approaches of MLE and the method of moments.

\section{5- Conclusion}

The purpose of this study was to derive the Bayesian posterior distribution with the highest equitailed posterior density interval under two different loss functions for point estimation and constructing credible intervals for estimating the Poisson parameter with a gamma prior distribution. The performances of two Bayesian methods under either the squared-error loss function or the quadratic loss function were compared with the existing classical MLE, EB, and EB with bootstrapping approaches through Monte Carlo simulations.

When analyzing the Poisson parameter distribution, the Bayesian methods created under the squared-error and quadratic loss functions produced the most suitable estimates for small true parameter values $(\theta=0.5,1$, or 2$)$ by providing the lowest MSE values for point estimation for all cases of sample size. Moreover, they attained CPs close to the nominal 0.95 confidence level with the lowest ALs for a large sample size (50 or 100). Meanwhile, for all cases of sample size, the classical MLE approach obtained the lowest MSE values for point estimation for large true parameter values $(\theta=5,10$, or 20) and provided CPs close to or greater than 0.95 and slightly longer ALs than the Bayesian methods. In addition, although the EB estimation method based on exponential prior distribution did not achieve the best results, they were close to those of the best estimator in each case, and so it is a good alternative for point estimation and confidence interval construction.

\section{6- Declarations}

\section{6-1-Data Availability Statement}

The data presented in this study are available on request from the corresponding author.

\section{6-2- Funding}

This research was supported in part by Research and Development Institute, Phetchabun Rajabhat University, Thailand.

\section{6-3-Conflicts of Interest}

The author declare that there is no conflict of interests regarding the publication of this manuscript. In addition, the ethical issues, including plagiarism, informed consent, misconduct, data fabrication and/or falsification, double publication and/or submission, and redundancies have been completely observed by the authors. 


\section{7- References}

[1] Araveeporn, Autcha. "Parameter Estimation of Poisson Distribution by Using Maximum Likelihood, Markov Chain Monte Carlo, and Bayes method." Science \& Technology Asia 19, no. 3 (2014): 1-14.

[2] Hassan, Anwar, Peer Bilal Ahmad, and M. Ishaq Bhatti. "On the Bayes Estimator of Parameter and Reliability Function of the Zero-Truncated Poisson Distribution”. Journal of the Korea Society for Industrial and Applied Mathematics 12, no.2, (January 2008): 97-108.

[3] Howlader, Hatem A., and Uditha Balasooriya. "Bayesian Estimation of the Distribution Function of the Poisson Model." Biometrical Journal 45, no. 7 (October 2003): 901-912. doi:10.1002/bimj.200390057.

[4] Takada, Yoshikazu. "Bayes Sequential Estimation of Poisson Mean Under A Linex Loss Function.” Sequential Analysis 20, no. 1-2 (May 31, 2001): 55-64. doi:10.1081/sqa-100102646.

[5] Hwang, Leng-Cheng, and Cheng-Hung Lee. "Bayes Sequential Estimation for a Poisson Process Under a LINEX Loss Function." Statistics 47, no. 4 (August 2013): 672-687. doi:10.1080/02331888.2011.648640.

[6] Lee, Cheng-Hung, and Leng-Cheng Hwang. “Asymptotic Optimal Estimation of Poisson Mean under LINEX Loss Function.” Communications in Statistics - Theory and Methods 40, no. 23 (December 2011): 4308-4321. doi:10.1080/03610926.2010.510252.

[7] Srivastava, Uma. "Bayesian Estimation of Shift Point in Poisson Model under Asymmetric Loss Functions." Pakistan Journal of Statistics and Operation Research 8, no. 1 (January 3, 2012): 31. doi:10.18187/pjsor.v8i1.306.

[8] Okasha, Hassan M., and Jianhua Wang. "E-Bayesian Estimation for the Geometric Model Based on Record Statistics.” Applied Mathematical Modelling 40, no. 1 (January 2016): 658-670. doi:10.1016/j.apm.2015.05.004.

[9] Han, Ming. "E-Bayesian Estimation of the Reliability Derived from Binomial Distribution." Applied Mathematical Modelling 35, no. 5 (May 2011): 2419-2424. doi:10.1016/j.apm.2010.11.051.

[10] Han, Ming. "The E-Bayesian and Hierarchical Bayesian Estimations of Pareto Distribution Parameter under Different Loss Functions." Journal of Statistical Computation and Simulation 87, no. 3 (August 18, 2016): 577-593. doi:10.1080/00949655.2016.1221408.

[11] Han, Ming. "E-Bayesian Estimation of the Exponentiated Distribution Family Parameter under LINEX Loss Function." Communications in Statistics - Theory and Methods 48, no. 3 (January 5, 2018): 648-659. doi:10.1080/03610926.2017.1417432.

[12] Hassan, M.R. and A.R. Baizid. "Bayesian Estimation under different Loss Functions Using Gamma Prior for the Case of Exponential Distribution”. Journal of Scientific Research 9, no.1, (2016): 67-78.

[13] Hassan, M.R. "A Bayesian Approach for Estimating the Scale Parameter of Double Exponential Distribution under Symmetric and Asymmetric Loss Functions". International Journal of Science and Research 8, (March 2019): 351-356.

[14] Naji, Loaiy F., and Huda A. Rasheed. "Bayesian Estimation for Two Parameters of Gamma Distribution Under Generalized Weighted Loss Function.” Iraqi Journal of Science 60, no. 5 (May 26, 2019): 1161-1171. doi:10.24996/ijs.2019.60.5.24.

[15] Naji, Loaiy F., and Huda Abdullah Rasheed. "Bayesian Estimation for Two Parameters of Gamma Distribution under Precautionary Loss Function.” Ibn Al-Haitham Journal for Pure and Applied Science 32, no. 1 (February 10, 2019 ): 193. doi:10.30526/32.1.1914.

[16] Naji, Loaiy F., and Huda A. Rasheed. "Estimate the Two Parameters of Gamma Distribution Under Entropy Loss Function." Iraqi Journal of Science (2019): 127-134.

[17] Okasha, Hassan, and Abdelfattah Mustafa. "E-Bayesian Estimation for the Weibull Distribution Under Adaptive Type-I Progressive Hybrid Censored Competing Risks Data.” Entropy 22, no. 8 (August 17, 2020): 903. doi:10.3390/e22080903.

[18] Basheer, Abdulkareem M., H. M. Okasha, A. H. El-Baz, and A. M. K. Tarabia. "E-Bayesian and Hierarchical Bayesian Estimations for the Inverse Weibull Distribution.” Annals of Data Science (January 9, 2021). doi:10.1007/s40745-020-00320$\mathrm{x}$.

[19] Athirakrishnan, R. B., and E. I. Abdul-Sathar. "E-Bayesian and Hierarchical Bayesian Estimation of Inverse Rayleigh Distribution." American Journal of Mathematical and Management Sciences (April 30, 2021): 1-22. doi:10.1080/01966324.2021.1914250.

[20] Yosboonruang, Noppadon, Sa-aat Niwitpong, and Suparat Niwitpong. "Measuring the Dispersion of Rainfall Using Bayesian Confidence Intervals for Coefficient of Variation of Delta-Lognormal Distribution: a Study from Thailand.” PeerJ 7 (July 22 , 2019): e7344. doi:10.7717/peerj.7344.

[21] Maneerat, Patcharee, Sa-aat Niwitpong, and Suparat Niwitpong. "A Bayesian Approach to Construct Confidence Intervals for Comparing the Rainfall Dispersion in Thailand.” PeerJ 8 (February 11, 2020): e8502. doi:10.7717/peerj.8502.

[22] Thangjai, Warisa, Sa-Aat Niwitpong, and Suparat Niwitpong. "Bayesian Confidence Intervals for Coefficients of Variation of PM10 Dispersion." Emerging Science Journal 5, no. 2 (April 2021): 139-154. doi:10.28991/esj-2021-01264. 
[23] Okasha, Hassan M. "E-Bayesian Estimation for the Lomax Distribution Based on Type-II Censored Data." Journal of the Egyptian Mathematical Society 22, no. 3 (October 2014): 489-495. doi:10.1016/j.joems.2013.12.009.

[24] Ijaz, M. "Bayesian Estimation of the Shape Parameter of Lomax Distribution under Uniform and Jeffery Prior with Engineering Applications". Gazi University Journal of Science, 34 (2021): 562-577.

[25] Yadav, Abhimanyu Singh, S. K. Singh, and Umesh Singh. "Bayesian estimation of $R=P[Y<X]$ for inverse Lomax distribution under progressive type-II censoring scheme." International Journal of System Assurance Engineering and Management 10, no. 5 (June 26, 2019): 905-917. doi:10.1007/s13198-019-00820-x.

[26] Robbins, Herbert. "The empirical Bayes approach to statistical decision problems." The Annals of Mathematical Statistics 35 , no. 1 (March 1964): 1-20.

[27] Yadpirun Supharakonsakun and Katechan Jampachasri. "The Comparison of Confidence Interval Estimation Methods for Parameter Using Empirical Bayes Methods in Poisson Distributed Data." 11th Thai Conference on Statistics and Applied Statistics (May 2010).

[28] Mohammed, Heba S. "Empirical E-Bayesian Estimation for the Parameter of Poisson Distribution." AIMS Mathematics 6, no. 8 (May 2021): 8205-8220. doi:10.3934/math.2021475.

[29] Li, C. and H. Hao. "E-Bayesian estimation and hierarchical Bayesian estimation of Poisson distribution parameter under entropy loss function". International Journal of Applied Mathematics 49, (2019): 369-374.

[30] Zhang, Ying-Ying, Ze-Yu Wang, Zheng-Min Duan, and Wen Mi. "The empirical Bayes estimators of the parameter of the Poisson distribution with a conjugate gamma prior under Stein's loss function." Journal of Statistical Computation and Simulation 89, no. 16 (2019): 3061-3074. doi:10.1080/00949655.2019.1652606.

[31] Zhang, Ying-Ying, Teng-Zhong Rong, and Man-Man Li. "The Empirical Bayes Estimators of the Mean and Variance Parameters of the Normal Distribution with a Conjugate Normal-Inverse-Gamma Prior by the Moment Method and the MLE Method." Communications in Statistics-Theory and Methods 48, no. 9 (Feb. 2019): 2286-2304. doi:10.1080/03610926.2018.1465081. 